\title{
A Scalable Optimization Mechanism for Pairwise based Discrete Hashing
}

\author{
Xiaoshuang Shi, Fuyong Xing, Zizhao Zhang, Manish Sapkota, Zhenhua Guo, and Lin Yang
}

\begin{abstract}
Maintaining the pair similarity relationship among originally high-dimensional data into a low-dimensional binary space is a popular strategy to learn binary codes. One simiple and intutive method is to utilize two identical code matrices produced by hash functions to approximate a pairwise real label matrix. However, the resulting quartic problem is difficult to directly solve due to the nonconvex and non-smooth nature of the objective. In this paper, unlike previous optimization methods using various relaxation strategies, we aim to directly solve the original quartic problem using a novel alternative optimization mechanism to linearize the quartic problem by introducing a linear regression model. Additionally, we find that gradually learning each batch of binary codes in a sequential mode, i.e. batch by batch, is greatly beneficial to the convergence of binary code learning. Based on this significant discovery and the proposed strategy, we introduce a scalable symmetric discrete hashing algorithm that gradually and smoothly updates each batch of binary codes. To further improve the smoothness, we also propose a greedy symmetric discrete hashing algorithm to update each bit of batch binary codes. Moreover, we extend the proposed optimization mechanism to solve the non-convex optimization problems for binary code learning in many other pairwise based hashing algorithms. Extensive experiments on benchmark single-label and multi-label databases demonstrate the superior performance of the proposed mechanism over recent state-of-the-art methods.
\end{abstract}

\section{INTRODUCTION}

Hashing has become a popular tool to tackle large-scale tasks in information retrieval, computer vision and machine leaning communities, since it aims to encode originally high-dimensional data into a variety of compact binary codes with maintaining the similarity between neighbors, leading to significant gains in both computation and storage [1] [2] [3].

Early endeavors in hashing focus on data-independent algorithms, like locality sensitive hashing (LSH) [4] and min-wise hashing (MinHash) [5] [6]. They construct hash functions by using random projections or permutations. However, due to randomized hashing, in practice they usually require long bits to achieve high precision per hash table and multiple tables to boost the recall [2]. To learn compact binary codes, data-dependent algorithms using available training data to learn hash functions have attracted increasing attention. Based on whether utilizing semantic label information, data-dependent algorithms can be categorized into two main groups: unsupervised and supervised. Unlike unsupervised hashing [7] [8] [9] that explores data intrinsic structures to preserve similarity relations between neighbors without any supervision, supervised hashing [10] [11] [12] employs semantic information to learn hash functions, and thus it usually achieves better retrieval accuracy than unsupervised hashing on semantic similarity measures.

X. Shi and L. Yang are with the J. Crayton Pruitt Family Department of Biomedical Engineering, University of Florida, Gainesville, FL, USA, e-mail: (xsshi2015@ufl.edu,lin.yang@bme.ufl.edu).

F. Xing and M. Sapkota is with the Department of Electrical and Computer Engineering, University of Florida, Gainesville, FL, USA, e-mail: ( $f$. xing@ufl.edu and manish.sapkota@gmail.com).

Z. Zhang is with the Department of Computer Science and Engineering, University of Florida, Gainesville, FL, USA, e-mail: (mr.zizhaozhang@gmail.com). Z. Guo is with Graduate School at Shenzhen, Tsinghua University, Shenzhen, Guodong, China, e-mail: (zhenhua.guo@sz.tsinghua.edu.cn).
Among supervised hashing algorithms, pairwise based hashing, maintaining the relationship of similar or dissimilar pairs in a Hamming space, is one popular method to exploit label information. Numerous pairwise based algorithms have been proposed in the past decade, including spectral hashing (SH) [7], linear discriminant analysis hashing (LDAHash) [12], minimal loss hashing (MLH) [10], binary reconstruction embedding (BRE) [8] and kernelbased supervised hashing (KSH) [13], etc. Although these algorithms have been demonstrated effective in many largescale tasks, their employed optimization strategies are usually insufficient to explore the similarity information defined in the non-convex and non-differential objective functions. In order to handle these non-smooth and non-convex problems, four main strategies have been proposed: symmetric/asymmetric relaxation, and asymmetric/symmetric discrete. Symmetric relaxation [7] [11] [13] [14] is to relax discrete binary vectors in a continuous feasible region followed by thresholding to obtain binary codes. Although symmetric relaxation can simplify the original optimization problem, it often generates large accumulated errors between hash and linear functions. To reduce the accumulated error, asymmetric relaxation [15] utilizes the element-wise product of discrete and its relaxed continuous matrices to approximate a pairwise label matrix. Asymmetric discrete hashing [16] [17] [18] usually utilizes the product of two distinct discrete matrices to preserve pair relations into a binary space. Symmetric discrete hashing [19] [20] firstly learns binary codes with preserving symmetric discrete constraints and then trains classifiers based on the learned discrete codes. Although most of hashing algorithms with these four strategies have achieved promising performance, they have at least one of the following four major disadvantages: (i) Learning binary codes employs relaxation and thresholding strategies, thereby producing large accumulated errors; (ii) Learning binary codes requires high storage and computation costs, 
i.e. $\mathcal{O}\left(n^{2}\right)$, where $n$ is the number of data points, thereby limiting their applications to large-scale tasks; (iii) The used pairwise label matrix usually emphasizes the difference of images among different classes but neglects their relevance within the same class. Hence, existing optimization methods might perform poorly to preserve the relevance information among images; (iv) The employed optimization methods focus on one type of optimization problems and it is difficult to directly apply them to other problems.

Motivated by aforementioned observations, in this paper, we propose a novel simple, general and scalable optimization method that can solve various pairwise based hashing models for directly learning binary codes. The main contributions are summarized as follows:

- We propose a novel alternative optimization mechanism to reformulate one typical quartic problem, in term of hash functions in the original objective of $\mathrm{KSH}$ [13], into a linear problem by introducing a linear regression model.

- We present and analyze a significant discovery that gradually updating each batch of binary codes in a sequential mode, i.e. batch by batch, is greatly beneficial to the convergence of binary code learning.

- We propose a scalable symmetric discrete hashing algorithm with gradually updating each batch of one discrete matrix. To make the update step more smooth, we further present a greedy symmetric discrete hashing algorithm to greedily update each bit of batch discrete matrices. Then we demonstrate that the proposed greedy hashing algorithm can be used to solve other optimization problems in pairwise based hashing.

- Extensive experiments on three benchmark databases: CIFAR-10 [21], NUS-WIDE [22] and COCO [23], demonstrate the superior performance of the proposed method over recent state of the arts, with low time costs.

\section{Related Work}

Based on the manner of using the label information, supervised hashing can be classified into three major categories: point-wise, multi-wise and pairwise.

Point-wise based hashing formulates the searching into one classification problem based on the rule that the classification accuracy with learned binary codes should be maximized. Supervised discrete hashing (SDH) [24] leverages one linear regression model to generate optimal binary codes. Fast supervised discrete hashing (FSDH) [25] improves the computation requirement of SDH via fast $\mathrm{SDH}$ approximation. Supervised quantization hashing (SQH) [26] introduces composite quantization into a linear model to further boost the discriminative ability of binary codes. Deep learning of binary hash codes (DLBHC) [27] and deep supervised convolutional hashing (DSCH) [28] employ convolutional neural network to simultaneously learn image representations and hash codes in a point-wised manner. Point-wise based hashing is scalable and its optimization problem is relatively easier than multi-wise and pairwise based hashing; however, its rule is inferior compared to the other two types of supervised hashing.
Multi-wise based hashing is also named as ranking based hashing that learns hash functions to maximize the agreement of similarity orders over two items between original and Hamming distances. Triplet ranking hashing (TRH) [29] and column generation hashing (CGH) [30] utilize a triplet ranking loss to maximumly preserve the similarity order. Order preserving hashing $(\mathrm{OPH})$ [31] learns hash functions to maximumly preserve the similarity order by taking it as a classification problem. Ranking based supervised hashing (RSH) [32] constructs a ranking triplet matrix to maintain orders of ranking lists. Ranking preserving hashing (RPH) [33] learns hash functions by directly optimizing a ranking measure, Normalized Discounted Cumulative Gain (NDCG) [34]. Top rank supervised binary coding (Top-RSBC) [35] focuses on boosting the precision of top positions in a Hamming distance ranking list. Discrete semantic ranking hashing (DSeRH) [36] learns hash functions to maintain ranking orders with preserving symmetric discrete constraints. Deep network in network hashing (DNNH) [37], deep semantic ranking hashing (DSRH) [38] and triplet-based deep binary embedding (TDBE) [39] utilize convolutional neural network to learn image representations and hash codes based on the triplet ranking loss over three items. Most of these multi-wise based hashing algorithms relax the ranking order or discrete binary codes in a continuously feasible region to solve their original nonconvex and non-smooth problems.

Pairwise based hashing maintains relationship among originally high-dimensional data into a Hamming space by calculating and preserving the relationship of each pair. $\mathrm{SH}$ [7] constructs one graph to maintain the similarity among neighbors and then utilizes it to map the high-dimensional data into a low-dimensional Hamming space. Although the original version of $\mathrm{SH}$ is unsupervised hashing, it is easily converted into a supervised algorithm. Inspired by $\mathrm{SH}$, many variants including anchor graph hashing [40], elastic embedding [41], discrete graph hashing (DGH) [2], and asymmetric discrete graph hashing (ADGH) [18] have been proposed. LDAHash [12] projects the high-dimensional descriptor vectors into a low-dimensional Hamming space with maximizing the distance of inter-class data and meanwhile minimizing the intra-class distances. MLH [10] adopts a structured prediction with latent variables to learn hash functions. BRE [8] aims to minimize the difference between Euclidean distances of original data and their Hamming distances. It leverages a coordinate-descent algorithm to solve the optimization problem with preserving symmetric discrete constraints. SSH [11] introduces a pairwise matrix and $\mathrm{KSH}$ [13] leverages the Hamming distance between pairs to approximate the pairwise matrix. This objective function is intuitive and simple, but the optimization problem is highly non-differential and difficult to directly solve. KSH utilizes a "symmetric relaxation + greedy" strategy to solve the problem. Two-step hashing (TSH) [14] and FastHash [42] relax the discrete constraints into a continuous region $[-1,1]$. Kernel based discrete supervised hashing $(\mathrm{KSDH})$ [15] adopts asymmetric relaxation to simultaneously learn the discrete matrix and a low-dimensional projection matrix for hash functions. Lin: Lin and Lin: V [16], asymmetric inner-product binary coding (AIBC) [17] and asymmetric discrete graph hashing (ADGH) [18] employ the asymmet- 
ric discrete mechanism to learn low-dimensional matrices. Column sampling based discrete supervised hashing (COSDISH) [20] adopts the column sampling strategy same as latent factor hashing (LFH) [43] but directly learn binary codes by reformulating the binary quadratic programming $(\mathrm{BQP})$ problems into equivalent clustering problems. Convolutional neural network hashing (CNNH) [19] divide the optimization problem into two sub-problems [44]: (i) learning binary codes by a coordinate descent algorithm using Newton directions; (ii) training a convolutional neural network using the learned binary codes as labels. After that, deep hashing network (DHN) [45] and deep supervised pairwise hashing (DSPH) [46] simultaneously learn image representations and binary codes using pairwise labels. HashNet [47] learns binary codes from imbalanced similarity data. Deep cauchy hashing (DCH) [48] utilizes pairwise labels to generate compact and concentrated binary codes for efficient and effective Hamming space retrieval. Unlike previous work, in this paper we aim to present a simpler, more general and scalable optimization method for binary code learning.

\section{Symmetric Discrete hashing via A Pair- WISE MATRIX}

In this paper, matrices and vectors are represented by boldface uppercase and lowercase letters, respectively. For a matrix $\mathbf{X} \in \mathbb{R}^{n \times d}$, its $i$-th row and $j$-th column vectors are denoted as $\mathbf{x}_{i}$ and $\mathbf{x}^{j}$, respectively, and $x_{i j}$ is one entry at the $i$-th row and $j$-th column.

\subsection{Formulation}

$\mathrm{KSH}$ [13] is one popular pairwise based hashing algorithm, which can preserve pairs' relationship with using two identical binary matrices to approximate one pairwise real matrix. Additionally, it is a quartic optimization problem in term of hash functions, and thus more typical and difficult to solve than that only containing a quadratic term with respect to hash functions. Therefore, we first propose a novel optimization mechanism to solve the original problem in $\mathrm{KSH}$, and then extend the proposed method to solve other pairwise based hashing models.

Given $n$ data points $\mathbf{X}=\left[\mathbf{x}_{1}, \mathbf{x}_{2}, \cdots, \mathbf{x}_{n}\right] \in \mathbb{R}^{n \times d}$, suppose one pair $\left(\mathbf{x}_{i}, \mathbf{x}_{j}\right) \in \mathcal{M}$ when they are neighbors in a metric space or share at least one common label, and $\left(\mathbf{x}_{i}, \mathbf{x}_{j}\right) \in \mathcal{C}$ when they are non-neighbors in a metric space or have different class labels. For the single-label multi-class problem, the pairwise matrix $\mathbf{S} \in \mathbb{R}^{n \times n}$ is defined as [11]:

$$
s_{i j}=\left\{\begin{array}{cc}
1 & \left(x_{i}, x_{j}\right) \in \mathcal{M}, \\
-1 & \left(x_{i}, x_{j}\right) \in \mathcal{C}, \\
0 & \text { otherwise. }
\end{array}\right.
$$

For the multi-label multi-class problem, similar to [49], $\mathbf{S}$ can be defined as:

$$
s_{i j}=\left\{\begin{array}{cc}
r_{i j} & \left(x_{i}, x_{j}\right) \in \mathcal{M}, \\
\alpha & \left(x_{i}, x_{j}\right) \in \mathcal{C}, \\
0 & \text { otherwise },
\end{array}\right.
$$

where $r_{i j}>0$ is the relevance between $\mathbf{x}_{i}$ and $\mathbf{x}_{j}$, which is defined as the number of common labels shared by $\mathbf{x}_{i}$ and $\mathbf{x}_{j} . \alpha<0$ is the weight to describe the difference between $\mathbf{x}_{i}$ and $\mathbf{x}_{j}$. In this paper, to preserve the difference between non-neighbor pairs, we empirically set $\alpha=-\frac{r_{\max }}{2}$, where $r_{\max }$ is the maximum relevance among all neighbor pairs. We do not set $\alpha=-r_{\max }$ because few data pairs have the relevance being $r_{\max }$.

To encode one data point $\mathbf{x} \in \mathbb{R}^{d}$ into $m$-bit hash codes, its $k$-th hash function can be defined as:

$$
h_{k}(\mathbf{x})=\operatorname{sgn}\left(\mathbf{x a}_{k}^{T}+b_{k}\right)
$$

where $\mathbf{a}_{k} \in \mathbb{R}^{d}$ is a projection vector, and $\operatorname{sgn}\left(\mathbf{x}_{i} \mathbf{a}_{k}^{T}+b_{k}\right)=1$ if $\mathbf{x}_{i} \mathbf{a}_{k}^{T}+b_{k} \geq 0$, otherwise $\operatorname{sgn}\left(\mathbf{x}_{i} \mathbf{a}_{k}^{T}+b_{k}\right)=-1$. Note that since $\mathbf{x a}_{k}^{T}+b_{k}$ can be written as the form $\mathbf{x a}_{k}^{T}$ with $\mathbf{x}$ adding one dimension and $\mathbf{a}_{k}$ absorbing $b_{k}$, for simplicity we utilize $h_{k}(\mathbf{x})=\operatorname{sgn}\left(\mathbf{x a}_{k}^{T}\right)$ in this paper. Let $\operatorname{code}_{m}(\mathbf{x})=$ $\left\{h_{1}(\mathbf{x}), h_{2}(\mathbf{x}), \cdots, h_{m}(\mathbf{x})\right\}$ be hash codes of $\mathbf{x}$, and then for any pair $\left(\mathbf{x}_{i}, \mathbf{x}_{j}\right)$, we have $m \geq \operatorname{code}_{m}\left(\mathbf{x}_{i}\right) \circ \operatorname{code}_{m}\left(\mathbf{x}_{j}\right) \geq$ $-m$. To approximate the pairwise matrix $\mathbf{S}$, same as [13], a least-squares style objective function is defined as:

$$
\min _{\mathbf{A}}\left\|\mathbf{H H}^{T}-\lambda \mathbf{S}\right\|_{F}^{2}, \text { s.t. } \mathbf{H}=\operatorname{sgn}\left(\mathbf{X A}^{T}\right),
$$

where $\lambda=\frac{m}{r_{\max }}$ and $\mathbf{A} \in \mathbb{R}^{m \times d}$ is a low-dimensional projection matrix. Eq. (4) is a quartic problem in term of hash functions, and this can be demonstrated by expanding its objective function.

\subsection{Symmetric Discrete Hashing}

\subsubsection{Formulation transformation}

In this subsection, we show the procedure to transform Eq. (4) into a linear problem. Since the objective function in Eq. (4) is a highly non-differential quartic problem in term of hash functions $\operatorname{sgn}\left(\mathbf{X} \mathbf{A}^{T}\right)$, it is difficult to directly solve this problem. Here, we solve the problem in Eq. (4) via a novel alternative optimization mechanism: reformulating the quartic problem in term of hash functions into a quadratic one and then linearizing the quadratic problem. We present the detailed procedure in the following.

Firstly, we introduce a Lemma to show one of our main motivations to transform the quartic problem into a linear problem.

Lemma 1. When the matrix $\mathbf{A} \in \mathbb{R}^{m \times d}$ satisfies the condition: $\mathbf{X A}^{T}=\mathbf{Y}$, it is a global solution of the following problem:

$$
\max _{\mathbf{A}} \operatorname{Tr}\left\{\mathbf{H}^{T} \mathbf{Y}\right\} \text {, s.t. } \mathbf{H}=\operatorname{sgn}\left(\mathbf{X} \mathbf{A}^{T}\right) .
$$

Lemma 1 is easy to solve, because when $\mathbf{X A} \mathbf{A}^{T}=\mathbf{Y}$, $\mathbf{H}=\operatorname{sgn}\left(\mathbf{X A}^{T}\right)=\operatorname{sgn}(\mathbf{Y})$ makes the objective in Eq. (5) attain the maximum. Since $\mathbf{A}$ satisfying $\mathbf{X} \mathbf{A}^{T}=\mathbf{Y}$ is a global solution of the problem in Eq. (5), it suggests that the problem in term of hash functions can be transformed into a linear problem in term of $\mathbf{A}$. Inspired by this observation, we can solve the quartic problem in term of hash functions. For brevity, in the following we first ignore the constraint $\mathbf{H}=\operatorname{sgn}\left(\mathbf{X A}^{T}\right)$ in Eq. (4) and aim to transform the quartic problem in term of $\mathbf{H}$ into the linear form as the objective in Eq. (5), and then obtain the low-dimensional projection matrix $\mathbf{A}$.

To reformulate the quartic problem in term of $\mathbf{H}$ into a quadratic one, in the $l$-th iteration, we set one discrete matrix to be $\mathbf{H}_{l-1}$ and aim to solve the following quadratic problem in term of $\mathbf{H}$ :

$$
\min _{\mathbf{H}}\left\|\mathbf{H}_{l-1} \mathbf{H}^{T}-\lambda \mathbf{S}\right\|_{F}^{2} \text {, s.t. } \mathbf{H} \in\{-1,1\}^{n \times m} .
$$


Note that the problem in Eq. (6) is not strictly equal to the problem in Eq. (4) w.r.t $\mathbf{H}$. However, when $\mathbf{H}_{l}=\mathbf{H}_{l-1}$, it is the optimal solution of both Eq. (6) and Eq. (4) w.r.t H. The details are shown in Proposition 1.

Proposition 1. When $\mathbf{H}_{l}=\mathbf{H}_{l-1}$, the optimal solution of Eq. (6) is also the optimal solution of Eq. (4) w.r.t $\mathbf{H}$.

Proof. Obviously, if $\mathbf{H}_{l}=\mathbf{H}_{l-1}$, it is the optimal solution of Eq. (6). Then we can consider the following formulation:

$$
\min _{\mathbf{H}_{l}, \mathbf{H}_{l-1}}\left\|\mathbf{H}_{l} \mathbf{H}_{l-1}-\mathbf{S}\right\|_{F}^{2} \leq \min _{\mathbf{H}}\|\mathbf{H H}-\mathbf{S}\|_{F}^{2}
$$

Similar to one major motivation of asymmetric discrete hashing algorithms [16] [18], in Eq. (7), the feasible region of $\mathbf{H}_{l}, \mathbf{H}_{l-1}$ in the left term is more flexible than $\mathbf{H}$ in the right term (Eq. (4)), i.e. the left term contains both two cases $\mathbf{H}_{l} \neq \mathbf{H}_{l-1}$ and $\mathbf{H}_{l}=\mathbf{H}_{l-1}$. Only when $\mathbf{H}_{l}=\mathbf{H}_{l-1}$, $\min _{\mathbf{H}_{l}, \mathbf{H}_{l-1}}\left\|\mathbf{H}_{l} \mathbf{H}_{l-1}-\mathbf{S}\right\|_{F}^{2}=\min _{\mathbf{H}}\|\mathbf{H H}-\mathbf{S}\|_{F}^{2}$. It suggests that when $\mathbf{H}_{l}=\mathbf{H}_{l-1}$, it is the optimal solution of Eq. (4). Therefore, when $\mathbf{H}_{l}=\mathbf{H}_{l-1}$, it is the optimal solution of both Eq. (4) and Eq. (6).

Inspired by Proposition 1, we aim to seek $\mathbf{H}_{l}=\mathbf{H}_{l-1}$ through solving the problem in Eq. (6). Because $\lambda$ is known and $\operatorname{Tr}\left\{\mathbf{S}^{T} \mathbf{S}\right\}=$ constant, the optimization problem in Eq. (6) equals:

$$
\begin{gathered}
\min _{\mathbf{H}} \operatorname{Tr}\left\{\mathbf{H H}_{l-1}^{T} \mathbf{H}_{l-1} \mathbf{H}^{T}\right\}-2 \lambda \operatorname{Tr}\left\{\mathbf{H H}_{l-1}^{T} \mathbf{S}\right\}, \\
\text { s.t. } \mathbf{H} \in\{-1,1\}^{n \times m} .
\end{gathered}
$$

Since $\operatorname{Tr}\left\{\mathbf{H H}_{l-1}^{T} \mathbf{S}\right\}$ is a linear problem in term of $\mathbf{H}$, the main difficulty to solve Eq. (8) is caused by the nonconvex quadratic term $\operatorname{Tr}\left\{\mathbf{H H}_{l-1}^{T} \mathbf{H}_{l-1} \mathbf{H}^{T}\right\}$. Thus we aim to linearize this quadratic term in term of $\mathbf{H}$ by introducing a linear regression model as follows:

Theorem 1. Given a discrete matrix $\mathbf{H} \in\{-1,1\}^{n \times m}$ and one real nonzero matrix $\mathbf{Z} \in \mathbb{R}^{m \times m}$ inf $\left\{\|\mathbf{H}-\mathbf{P Z}\|_{F}^{2}+\left\|\mathbf{P} \Gamma^{\frac{1}{2}}\right\|_{F}^{2} \mid \mathbf{P} \in \mathbb{R}^{n \times m}, \Gamma_{i i}>0\right\} \quad=$ $\operatorname{Tr}\left\{\mathbf{H}\left(\mathbf{I}_{m}-\mathbf{Z}^{T}\left(\mathbf{Z} \mathbf{Z}^{T}+\boldsymbol{\Gamma}\right)^{-1} \mathbf{Z}\right) \mathbf{H}^{T}\right\}$, where $\boldsymbol{\Gamma} \in \mathbb{R}^{m \times m}$ is a diagonal matrix and $\mathbf{I}_{m} \in \mathbb{R}^{m \times m}$ is an identity matrix.

Proof. It is easy to verify that $\mathbf{P}^{*}=\mathbf{H} \mathbf{Z}^{T}\left(\mathbf{Z} \mathbf{Z}^{T}+\mathbf{\Gamma}\right)^{-1}$ is the global optimal solution to the problem $\min _{\mathbf{P}}\|\mathbf{H}-\mathbf{P Z}\|_{F}^{2}+$ $\left\|\mathbf{P} \Gamma^{\frac{1}{2}}\right\|_{F}^{2}$. Substituting $\mathbf{P}^{*}$ into the above objective, its minimum value is $\operatorname{Tr}\left\{\mathbf{H}\left(\mathbf{I}_{m}-\mathbf{Z}^{T}\left(\mathbf{Z} \mathbf{Z}^{T}+\boldsymbol{\Gamma}\right)^{-1} \mathbf{Z}\right) \mathbf{H}^{T}\right\}$. Therefore, Theorem 1 is proved.

Theorem 1 suggests that when $\mathbf{H}_{l-1}^{T} \mathbf{H}_{l-1}=\gamma\left(\mathbf{I}_{m}-\right.$ $\left.\mathbf{Z}^{T}\left(\mathbf{Z} \mathbf{Z}^{T}+\boldsymbol{\Gamma}\right)^{-1} \mathbf{Z}\right)$, the quadratic problem in Eq. (8) can be linearized as a regression type. We show the details in Theorem 2.

Theorem 2. When $\mathbf{H}_{l-1}^{T} \mathbf{H}_{l-1}=\gamma\left(\mathbf{I}_{m}-\mathbf{Z}^{T}\left(\mathbf{Z} \mathbf{Z}^{T}+\boldsymbol{\Gamma}\right)^{-1} \mathbf{Z}\right)$, where $\gamma$ is a constant, the problem in Eq. (8) can be reformulated as:

$$
\begin{gathered}
\min _{\mathbf{H}, \mathbf{P}} \gamma\left(\|\mathbf{H}-\mathbf{P Z}\|_{F}^{2}+\left\|\mathbf{P} \boldsymbol{\Gamma}^{\frac{1}{2}}\right\|_{F}^{2}\right) \\
-2 \lambda \operatorname{Tr}\left\{\mathbf{H H}_{l-1}^{T} \mathbf{S}^{T}\right\} \text {, s.t. } \mathbf{H} \in\{-1,1\}^{n \times m} .
\end{gathered}
$$

Proof. Based on the condition $\mathbf{H}_{l-1}^{T} \mathbf{H}_{l-1}=\gamma\left(\mathbf{I}_{m}-\right.$ $\left.\mathbf{Z}^{T}\left(\mathbf{Z} \mathbf{Z}^{T}+\boldsymbol{\Gamma}\right)^{-1} \mathbf{Z}\right)$ and Theorem 1, substituting $\mathbf{P}^{*}=$
$\mathbf{H} \mathbf{Z}^{T}\left(\mathbf{Z} \mathbf{Z}^{T}+\boldsymbol{\Gamma}\right)^{-1}$ into the objective of Eq. (9), whose objective value is equal to that of Eq. (8), Therefore, Theorem 2 is proved.

Since $\operatorname{Tr}\left\{\mathbf{H}^{T} \mathbf{H}\right\}=m n$, the problem in Eq. (9) equals:

$$
\begin{gathered}
\max _{\mathbf{H}, \mathbf{P}} \operatorname{Tr}\left\{\mathbf{H}\left(\frac{\lambda}{\gamma} \mathbf{H}_{l-1}^{T} \mathbf{S}+\mathbf{Z}^{T} \mathbf{P}^{T}\right)\right\} \\
-\frac{1}{2}\left(\left\|\mathbf{P} \boldsymbol{\Gamma}^{\frac{1}{2}}\right\|_{F}^{2}+\operatorname{Tr}\left\{\mathbf{P} \mathbf{Z} \mathbf{Z}^{T} \mathbf{P}^{T}\right\}\right), \\
\text { s.t. } \mathbf{H} \in\{-1,1\}^{n \times m},
\end{gathered}
$$

which is a linear problem in term of $\mathbf{H}$.

Next, we demonstrate that there exists $\gamma$ and $\mathbf{Z}$ such that $\mathbf{H}_{l-1}^{T} \mathbf{H}_{l-1}=\gamma\left(\mathbf{I}_{m}-\mathbf{Z}^{T}\left(\mathbf{Z} \mathbf{Z}^{T}+\boldsymbol{\Gamma}\right)^{-1} \mathbf{Z}\right)$. The details are shown in Theorem 3.

Theorem 3. Suppose that a full rank matrix $\mathbf{H}_{l-1}^{T} \mathbf{H}_{l-1}=$ $\mathbf{U} \boldsymbol{\Lambda}^{2} \mathbf{U}^{T}$, where $\boldsymbol{\Lambda} \in \mathbb{R}^{m \times m}$ is a positive diagonal matrix and $\mathbf{U}^{T} \mathbf{U}=\mathbf{U U}^{T}=\mathbf{I}_{m}$. If $\gamma \geq \Lambda_{i i}^{2}$ and $\Gamma_{i i}>0$, $(1 \leq i \leq m)$ and a real nonzero matrix $\mathbf{Z}=\mathbf{V} \boldsymbol{\Delta} \mathbf{U}^{T}$ satisfies the conditions: $\mathbf{V}^{T} \mathbf{V}=\mathbf{V} \mathbf{V}^{T}=\mathbf{I}_{m}$, and $\boldsymbol{\Delta} \in \mathbb{R}^{m \times m}$ is a non-negative real diagonal matrix with the $i$-th diagonal element being $\Delta_{i i}=\sqrt{\frac{\gamma \Gamma_{i i}}{\Lambda_{i i}^{2}}-\Gamma_{i i}}$.

Proof. Based on singular value decomposition (SVD), there exist matrices $\mathbf{V}$ and $\mathbf{U}_{\mathbf{Z}}$, satisfying the conditions $\mathbf{V V}^{T}=$ $\mathbf{V}^{T} \mathbf{V}=\mathbf{I}_{m}$ and $\mathbf{U}_{\mathbf{Z}} \mathbf{U}_{\mathbf{Z}}^{T}=\mathbf{U}_{\mathbf{Z}}^{T} \mathbf{U}_{\mathbf{Z}}=\mathbf{I}_{m}$, such that a real nonzero matrix $\mathbf{Z}$ is represented by $\mathbf{Z}=\mathbf{V} \boldsymbol{\Delta} \mathbf{U}_{\mathbf{Z}}^{T}$, where $\boldsymbol{\Delta}$ is a non-negative real diagonal matrix. Then $\mathbf{I}_{m}-\mathbf{Z}^{T}\left(\mathbf{Z} \mathbf{Z}^{T}+\boldsymbol{\Gamma}\right)^{-1} \mathbf{Z}=\mathbf{U}_{\mathbf{Z}}\left(\mathbf{I}_{m}-\boldsymbol{\Delta}\left(\boldsymbol{\Delta}^{2}+\boldsymbol{\Gamma}\right)^{-1} \boldsymbol{\Delta}\right) \mathbf{U}_{\mathbf{Z}}^{T}$. Note that when the vectors in $\mathbf{V}$ and $\mathbf{U}_{\mathbf{Z}}$ corresponds to the zero diagonal elements, they can be constructed by employing a Gram-Schmidt process such that $\mathbf{V} \mathbf{V}^{T}=\mathbf{V}^{T} \mathbf{V}=\mathbf{I}_{m}$ and $\mathbf{U}_{\mathbf{Z}} \mathbf{U}_{\mathbf{Z}}^{T}=\mathbf{U}_{\mathbf{Z}}^{T} \mathbf{U}_{\mathbf{Z}}=\mathbf{I}_{m}$, and these constructed vectors are not unique.

Since $\mathbf{H}_{l-1}^{T} \mathbf{H}_{l-1}=\mathbf{U} \boldsymbol{\Lambda}^{2} \mathbf{U}^{T}$ and $\mathbf{I}_{m}-\mathbf{Z}^{T}\left(\mathbf{Z} \mathbf{Z}^{T}+\right.$ $\boldsymbol{\Gamma})^{-1} \mathbf{Z}=\frac{\mathbf{H}_{l-1}^{T} \mathbf{H}_{l-1}}{\gamma}$, it can have $\gamma \Gamma_{i i}\left(\Delta_{i i}^{2}+\Gamma_{i i}\right)^{-1}=\Lambda_{i i}^{2}$ when $\mathbf{U}_{\mathbf{Z}}=\mathbf{U}$. Since there exists $0<\Gamma_{i i}\left(\Delta_{i i}^{2}+\Gamma_{i i}\right)^{-1} \leq 1$, $\gamma$ should satisfy: $\gamma \geq \Lambda_{i i}^{2}$ and $\Gamma_{i i}>0$. Additionally, based on $\gamma \Gamma_{i i}\left(\Delta_{i i}^{2}+\Gamma_{i i}\right)^{-1}=\Lambda_{i i}^{2}$, there exists $\Delta_{i i}=\sqrt{\frac{\gamma \Gamma_{i i}}{\Lambda_{i i}^{2}}-\Gamma_{i i}}$. Therefore, Theorem 3 is proved.

$\mathbf{H}_{l-1}^{T} \mathbf{H}_{l-1}$ is usually a positive-definite matrix thanks to $m<<n$, leading to $\Lambda_{i i}>0$. Based on Theorem 3, it is easy to construct a real nonzero matrix $\mathbf{Z}$. Since $\gamma \geq \Lambda_{i i}^{2}$, we set $\gamma=\max _{i i}^{2}+\beta$ for simplicity, where $1 \leq i \leq m$ and $\beta \geq 0$ is a constant. Then Eq. (10) can be solved by alternatively updating $\mathbf{H}, \mathbf{Z}$ and $\mathbf{P}$. Actually, we can obtain $\mathbf{H}$ by using an efficient algorithm in Theorem 4 that does not need to compute the matrices $\mathbf{Z}$ and $\mathbf{P}$.

Theorem 4. For the inner $t$-th iteration embedded in the outer l-th iteration, the problem in Eq. (10) can be reformulated as the following problem:

$$
\begin{gathered}
\max _{\mathbf{H}_{l}} \operatorname{Tr}\left\{\mathbf { H } _ { l } \left(\left(\gamma \mathbf{I}_{m}-\mathbf{H}_{l-1}^{T} \mathbf{H}_{l-1}\right) \mathbf{H}_{l_{t-1}}^{T}\right.\right. \\
\left.\left.+\lambda \mathbf{H}_{l-1}^{T} \mathbf{S}\right)\right\}, \text { s.t. } \mathbf{H}_{l} \in\{-1,1\}^{n \times m},
\end{gathered}
$$

where $\mathbf{H}_{l} \in \mathbb{R}^{n \times m}$ denotes binary codes $\mathbf{H}$ in the outer l-th iteration, and $\mathbf{H}_{l_{t-1}}$ represents the obtained binary codes $\mathbf{H}$ at the inner $t-1$-th iteration embedded in the outer $l$-th iteration. 


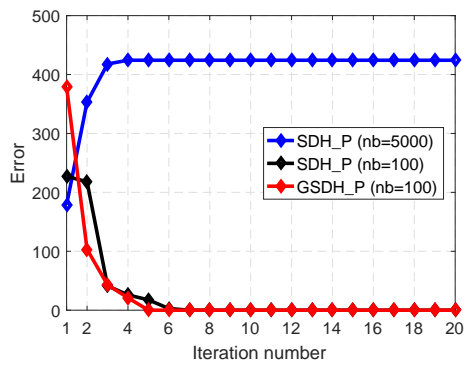

(a) @ $\left\|\mathbf{H}_{l}-\mathbf{H}_{l-1}\right\|_{F}$

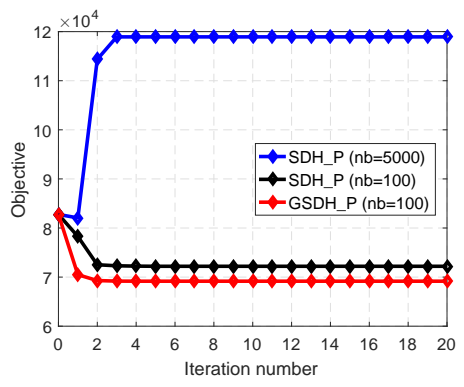

(b) @ $\left\|\mathbf{H} \mathbf{H}^{T}-\lambda \mathbf{S}\right\|_{F}$

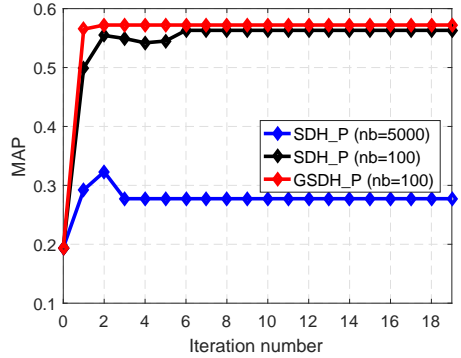

(c) @ Top 500

Fig. 1. The error, objective and mean average precision (MAP) of SDH_P and GSDH_P with different number of iterations. (In total we select 5K training and $1 \mathrm{~K}$ query images from the CIFAR10 database, and employ all training samples as anchors. In (b) and (c), when the number of iteration is 0 , the results are achieved by using the projection matrix calculated in the initialization step.)

Proof. In Eq. (10), for the inner $t$-th iteration embedded in the outer $l$-th iteration, fixing $\mathbf{H}$ as $\mathbf{H}_{l_{t-1}}$, it is easy to obtain $\mathbf{P}_{l_{t}}=\mathbf{H}_{l_{t-1}} \mathbf{Z}^{T}\left(\mathbf{Z} \mathbf{Z}^{T}+\boldsymbol{\Gamma}\right)^{-1}$. Substituting $\mathbf{P}_{l_{t}}$ into Eq. (10), it becomes:

$$
\begin{gathered}
\max _{\mathbf{H}_{l}} \operatorname{Tr}\left\{\mathbf{H}_{l} \mathbf{Z}^{T}\left(\mathbf{Z} \mathbf{Z}^{T}+\mathbf{\Gamma}\right)^{-1} \mathbf{Z} \mathbf{H}_{l_{t-1}}^{T}\right\} \\
+\frac{\lambda}{\gamma} \operatorname{Tr}\left\{\mathbf{H}_{l} \mathbf{H}_{l-1}^{T} \mathbf{S}\right\}, \text { s.t. } \mathbf{H}_{l} \in\{-1,1\}^{n \times m} .
\end{gathered}
$$

Based on Theorem 2 and its proof, there have $\gamma \mathbf{I}_{m}-$ $\mathbf{H}_{l-1}^{T} \mathbf{H}_{l-1}=\gamma \mathbf{Z}^{T}\left(\mathbf{Z} \mathbf{Z}^{T}+\boldsymbol{\Gamma}\right)^{-1} \mathbf{Z}$. Substituting it into Eq. (12), whose optimization problem becomes Eq. (11). Therefore, Theorem 4 is proved.

For the inner loop embedded in the outer $l$-th iteration, there are many choices for the initialization value $\mathbf{H}_{l_{0}}$. Here, we set $\mathbf{H}_{l_{0}}=\mathbf{H}_{l-1}$. At the $t$-th iteration, the global solution of Eq. (11) is $\mathbf{H}_{l_{t}}=\operatorname{sgn}\left(\lambda \mathbf{S}^{T} \mathbf{H}_{l-1}+\mathbf{H}_{l_{t-1}}\left(\gamma \mathbf{I}_{m}-\right.\right.$ $\left.\mathbf{H}_{l-1}^{T} \mathbf{H}_{l-1}\right)$ ). Additionally, for the inner loop, both $\mathbf{P}_{l_{t}}$ and $\mathbf{H}_{l_{t}}$ are global solutions of the $t$-th iteration, it suggests that the objective of Eq. (11) will be non-decreasing and converge to at least a local optima. Therefore, we have the following theorem.

Theorem 5. For the inner loop embedded in the outer l-th iteration, the objective of Eq. (11) is monotonically non-decreasing in each iteration and will converge to at least a local optima.

Although Theorem 5 suggests that the objective of Eq. (11) will converge, its convergence is largely affected by the parameter $\gamma$, which is used to balance the convergence and semantic information in $\mathbf{S}$. Usually, the larger $\gamma$, the faster convergence but the more loss of semantic information. Therefore, we empirically set a small non-negative constant for $\beta$, i.e. $0 \leq \beta \leq 100$, where $\gamma=\max \Lambda_{i i}^{2}+\beta$.

Based on Eq. (11), the optimal solution $\mathbf{H}_{l}^{*}$ can be obtained. Then we utilize $\mathbf{H}_{l}^{*}$ to replace $\mathbf{H}_{l-1}$ in Eq. (6) for next iteration in order to obtain the optimal solution $\mathbf{H}^{*}$. Since $\mathbf{H}^{*}=\operatorname{sgn}\left(\lambda \mathbf{S}^{T} \mathbf{H}^{*}+\mathbf{H}^{*}\left(\gamma \mathbf{I}_{m}-\mathbf{H}^{* T} \mathbf{H}^{*}\right)\right)$ and $\mathbf{H}=\operatorname{sgn}\left(\mathbf{X A}^{T}\right)$, based on Lemma 1, A should satisfy $\mathbf{X A}^{T}=\lambda \mathbf{S}^{T} \mathbf{H}^{*}+\mathbf{H}^{*}\left(\gamma \mathbf{I}_{m}-\mathbf{H}^{* T} \mathbf{H}^{*}\right)$. However, it is an overdetermined linear system due to $n>>d$. For simplicity, we utilize a least-squares model to obtain $\mathbf{A}$, which is $\mathbf{A}=\left(\lambda \mathbf{H}^{* T} \mathbf{S}+\left(\gamma \mathbf{I}_{m}-\mathbf{H}^{* T} \mathbf{H}^{*}\right) \mathbf{H}^{* T}\right) \mathbf{X}\left(\mathbf{X}^{T} \mathbf{X}\right)^{-1}$.

\subsubsection{Scalable symmetric discrete hashing with updating batch binary codes}

Remark: The optimal solution of Eq. (6) is at least the local optimal solution of Eq. (4) only when $\left\|\mathbf{H}_{l}-\mathbf{H}_{l-1}\right\|_{F}=0$.
Given an initialization $\mathbf{H}^{0}, \mathbf{H}$ can be alternatively updated by solving Eq. (11). However, with updating all binary codes at once on the non-convex feasible region, $\mathbf{H}$ might change on two different discrete matrices, which would lead to the error $\left\|\mathbf{H}_{l}-\mathbf{H}_{l-1}\right\|_{F} \neq 0$ (please see Figure 1a) and the objective of Eq. (4) becomes worse (please see Figure 1b). Therefore, we divide $\mathbf{H}$ into a variety of batches and gradually update each of them in a sequential mode, i.e. batch by batch.

To update one batch of $\mathbf{H}$, i.e. $\mathbf{H}_{b}=\mathbf{H}(i d x,:)$, where $i d x \in \mathbb{R}^{n_{b}}$ is one column vector denoting the index of selected binary codes in $\mathbf{H}$, the optimization problem derived from Eq. (6) is:

$$
\begin{gathered}
\min _{\mathbf{H}_{b}}\left\|\mathbf{H}_{l-1} \mathbf{H}_{b}^{T}-\lambda \mathbf{S}_{b}\right\|_{F}^{2}, \\
\text { s.t. } \mathbf{H}_{b} \in\{-1,1\}^{n_{b} \times m}, \mathbf{H}_{b} \subset \mathbf{H},
\end{gathered}
$$

where $\mathbf{S}_{b}=\mathbf{S}(:, i d x) \in \mathbb{R}^{n \times n_{b}}$.

Furthermore, although $\mathbf{S} \in \mathbb{R}^{n \times n}$ is high-dimensional for large $n$, it is low-rank or can be approximated as a lowrank matrix. Similar to previous algorithms [40] [18], we can select $p(p<<n)$ samples from $n$ training samples as anchors and then construct an anchor based pairwise matrix $\mathbf{S}_{A} \in \mathbb{R}^{p \times n}$, which preserves almost all similarity information of $\mathbf{S}$. Let $\mathbf{H}_{A} \in\{-1,1\}^{p \times m}$ denote binary codes of anchors, and then utilize $\mathbf{S}_{A}$ to replace $\mathbf{S}$ for updating $\mathbf{H}_{b}$, Eq. (13) becomes:

$$
\begin{gathered}
\min _{\mathbf{H}_{b}}\left\|\mathbf{H}_{A l-1} \mathbf{H}_{b}^{T}-\lambda \mathbf{S}_{A b}\right\|_{F}^{2}, \\
\text { s.t. } \mathbf{H}_{b} \in\{-1,1\}^{n_{b} \times m}, \mathbf{H}_{A} \subset \mathbf{H}, \mathbf{H}_{b} \subset \mathbf{H},
\end{gathered}
$$

where $\mathbf{H}_{A l-1}$ denotes $\mathbf{H}_{A}$ obtained at the $l-1$-th iteration in the outer loop, and $\mathbf{S}_{A b}=\mathbf{S}_{A}(:, i d x) \in \mathbb{R}^{p \times n_{b}}$.

Similar to Eq. (6), the problem in Eq. (14) can be firstly transformed into a quadratic problem, and then can be reformulated as a similar form to Eq. (11) based on Theorems 1-4. e.g.

$$
\begin{gathered}
\max _{\mathbf{H}_{b l}} \operatorname{Tr}\left\{\mathbf { H } _ { b l } \left(\left(\gamma \mathbf{I}_{m}-\mathbf{H}_{A l-1}^{T} \mathbf{H}_{A l-1}\right) \mathbf{H}_{b l_{t-1}}^{T}\right.\right. \\
\left.\left.+\lambda \mathbf{H}_{A l-1}^{T} \mathbf{S}_{A b}\right)\right\} \text {, s.t. } \mathbf{H}_{b l} \in\{-1,1\}^{n_{b} \times m} .
\end{gathered}
$$

where $\mathbf{H}_{b l}$ denotes the batch binary codes at the $l$-th iteration in the outer loop.

For clarity, we present the detailed optimization procedure to attain $\mathbf{H}$ by updating each batch $\mathbf{H}_{b}$ and calculate the projection matrix $\mathbf{A}$ in Algorithm 1, namely symmetric discrete hashing via a pairwise matrix (SDH_P). For Algorithm 1, with gradually updating each batch of $\mathbf{H}$, the error 


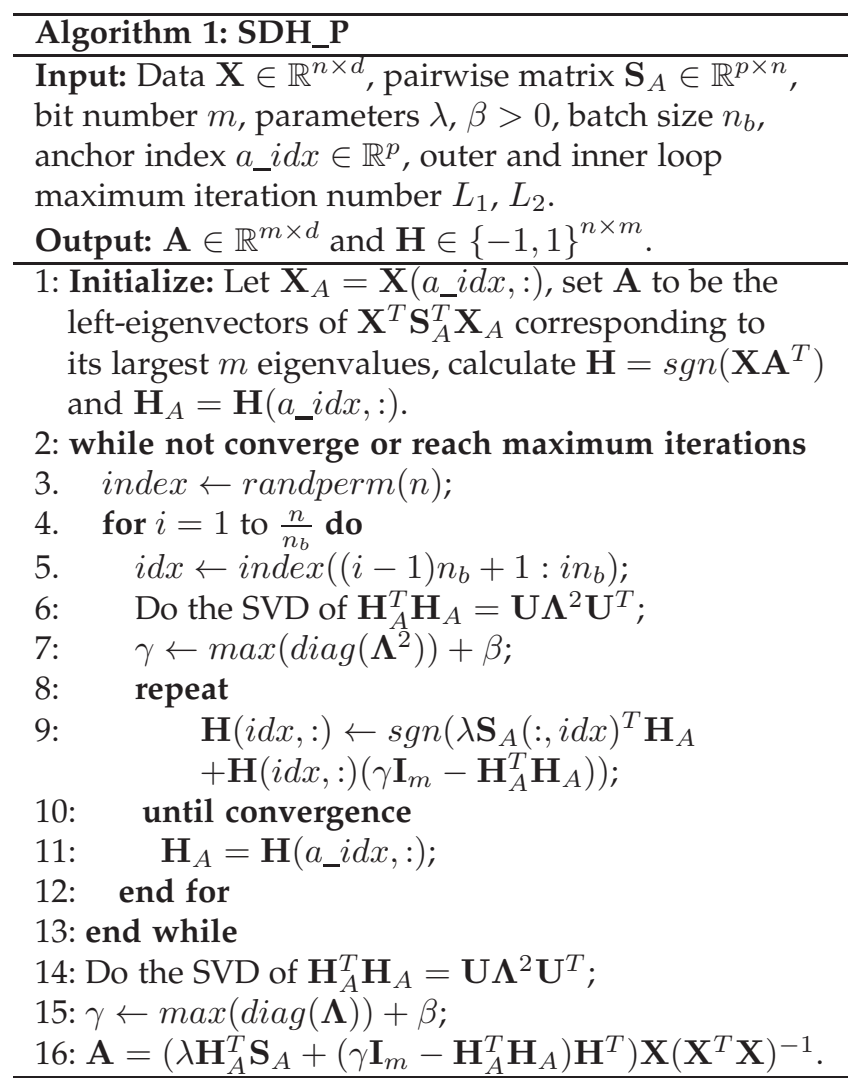

$\left\|\mathbf{H}_{l}-\mathbf{H}_{l-1}\right\|_{F}$ usually converges to zero (please see Figure 1a) and the objective of Eq. (4) also converges to a better local optima (please see Figure 1b). Besides, we also display the retrieval performance in term of mean average precision (MAP) with a small batch size and different iterations in Figure 1c.

\subsection{Greedy Symmetric Discrete Hashing}

To make the update step more smooth, we greedily update each bit of the batch matrix $\mathbf{H}_{b}$. Suppose that $\mathbf{h}_{b}^{j}=\mathbf{H}(i d x, j)$ is the $j$-th bit of $\mathbf{H}_{b}$, it can be updated by solving the following optimization problem:

$$
\begin{gathered}
\min _{\mathbf{h}_{b}^{j}}\left\|\mathbf{h}_{A}^{j} \mathbf{h}_{b}^{j T}-\lambda\left(\mathbf{S}_{A b}-\sum_{k \neq j}^{m} \mathbf{h}_{A}^{k} \mathbf{h}_{b}^{k T}\right)\right\|_{F}^{2}, \\
\text { s.t. } \mathbf{h}_{b}^{j} \in\{-1,1\}^{n_{b}}, \mathbf{h}_{A}^{j} \subset \mathbf{h}^{j}, \mathbf{h}_{b}^{j} \subset \mathbf{h}^{j},
\end{gathered}
$$

where $\mathbf{h}_{A}^{j}$ and $\mathbf{h}_{A}^{k}$ represent the $j$-th and $k$-th bits of $\mathbf{H}_{A}$, respectively, and $\mathbf{h}_{b}^{k}$ is the $k$-th bit of $\mathbf{H}_{b}$.

The problem in Eq. (16) can also be firstly transformed into a quadratic problem and then solved using Theorems 1-4. Similar to Eq. (11), the problem in Eq. (16) can be transformed to:

$$
\begin{aligned}
& \max _{\mathbf{h}_{b l}^{j}} \operatorname{Tr}\left\{\mathbf{h}_{b l}^{j}\left(\beta \mathbf{h}_{b l_{t-1}}^{j T}+\lambda \mathbf{h}_{A l-1}^{j T} \widetilde{\mathbf{S}}_{A b}\right)\right\}, \\
& \text { s.t. } \mathbf{h}_{b l}^{j} \in\{-1,1\}^{n_{b}},
\end{aligned}
$$

where $\mathbf{h}_{b l}^{j}$ is the $\mathbf{h}_{b}^{j}$ obtained at the $l$-th iteration in the outer loop, $\mathbf{h}_{b l_{t-1}}^{j}$ is the $\mathbf{h}_{b}^{j}$ obtained at the $t-1$-th iteration in inner loop embedded in the $l$-th outer loop and $\widetilde{\mathbf{S}}_{A b}=\mathbf{S}_{A b}-$ $\sum_{k \neq j}^{m} \mathbf{h}_{A}^{k} \mathbf{h}_{b}^{k T}$.

In summary, we show the detailed optimization procedure in Algorithm 2, namely greedy symmetric discrete hashing via a pairwise matrix (GSDH_P). The error

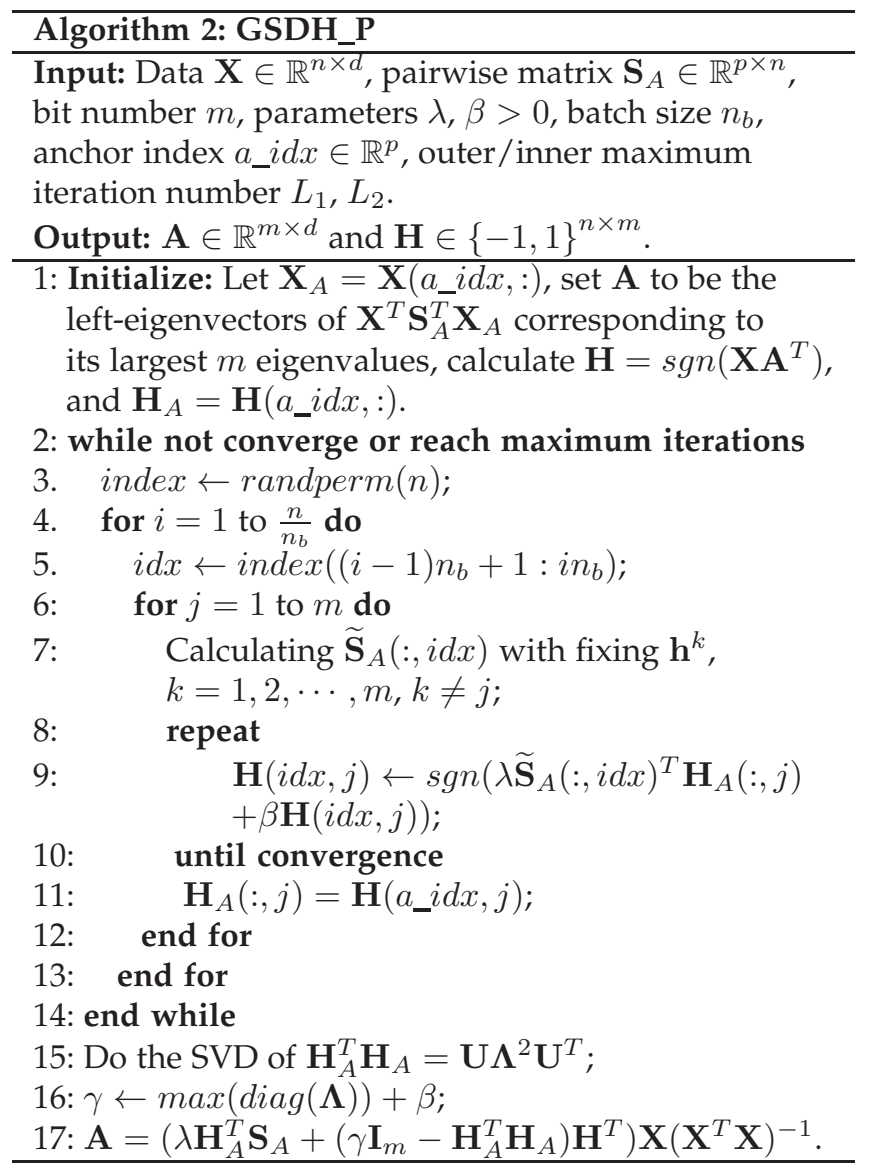

$\left\|\mathbf{H}_{l}-\mathbf{H}_{l-1}\right\|_{F}$ and the objective of Eq. (4) in Algorithm 2 and its retrieval performance in term of MAP with different number of iterations are shown in Figure $1 \mathrm{a}, \mathrm{b}$ and $\mathrm{c}$, respectively.

Out-of-sample: In the query stage, $\mathbf{H}$ is employed as the binary codes of training data. We adopt two strategies to encode the query data point $\mathbf{q} \in \mathbb{R}^{d}$ : (i) encoding it using $h(\mathbf{q})=\operatorname{sgn}\left(\mathbf{q A}^{T}\right)$; (ii) similar to previous algorithms [50] [19], employing $\mathbf{H}$ as labels to learn a classification model, like least-squares, decision trees (DT) or convolutional neural networks (CNNs), to classify q.

\subsection{Convergence Analysis}

Empirically, when $n>>n_{b}$, the proposed algorithms can converge to at least a local optima, although they cannot be theoretically guaranteed to converge in all cases. Here, we explain why gradually updating each batch of binary codes is beneficial to the convergence of hash code learning.

In Eq. (11), with updating one batch of $\mathbf{H}$, i.e. $\mathbf{H}_{b} \in$ $\{-1,1\}^{n_{b} \times m}$, Eq. (11) becomes:

$$
\begin{gathered}
\max _{\mathbf{H} b l} \operatorname{Tr}\left\{\mathbf { H } _ { b l } \left(\left(\gamma \mathbf{I}_{m}-\mathbf{H}_{l-1}^{T} \mathbf{H}_{l-1}\right) \mathbf{H}_{b l_{t-1}}^{T}\right.\right. \\
\left.\left.+\lambda \mathbf{H}_{l-1}^{T} \mathbf{S}_{b}\right)\right\}, \text { s.t. } \mathbf{H}_{b l} \in\{-1,1\}^{n_{b} \times m},
\end{gathered}
$$

The hash code matrix $\mathbf{H}$ can be represented as $\mathbf{H}=$ $\left[\mathbf{H}_{b} ; \tilde{\mathbf{H}}\right]$, where $\widetilde{\mathbf{H}} \in\{-1,1\}^{\left(n-n_{b}\right) \times m}$. Since $n>>n_{b}$, the objective of Eq. (18) is determined by:

$$
\begin{aligned}
& \max _{\mathbf{H}_{b l}} \operatorname{Tr}\left\{\mathbf { H } _ { b l } \left(\left(\gamma \mathbf{I}_{m}-\widetilde{\mathbf{H}}_{l-1}^{T} \widetilde{\mathbf{H}}_{l-1}\right) \mathbf{H}_{b l_{t-1}}^{T}\right.\right. \\
& \left.\left.+\lambda \widetilde{\mathbf{H}}_{l-1}^{T} \mathbf{S}_{b}\right)\right\}, \text { s.t. } \mathbf{H}_{b l} \in\{-1,1\}^{n_{b} \times m},
\end{aligned}
$$


Based on Theorem 5, the inner loop can theoretically guarantee the convergence of the objective in Eq. (11), and thus the optimal solution $\mathbf{H}_{b l}^{*}$ of Eq. (19) can be obtained by the inner loop. Then it has:

$$
\begin{aligned}
& \operatorname{Tr}\left\{\mathbf{H}_{b l}^{*}\left(\left(\gamma \mathbf{I}_{m}-\widetilde{\mathbf{H}}_{l-1}^{T} \widetilde{\mathbf{H}}_{l-1}\right) \mathbf{H}_{b l}^{* T}+\lambda \widetilde{\mathbf{H}}_{l-1}^{T} \mathbf{S}_{b}\right)\right\} \geq \\
& \operatorname{Tr}\left\{\mathbf{H}_{b l-1}\left(\left(\gamma \mathbf{I}_{m}-\widetilde{\mathbf{H}}_{l-1}^{T} \widetilde{\mathbf{H}}_{l-1}\right) \mathbf{H}_{b l-1}^{T}+\lambda \widetilde{\mathbf{H}}_{l-1}^{T} \mathbf{S}_{b}\right)\right\}
\end{aligned}
$$

Because of $n>>n_{b}$, Eq. (20) usually leads to

$$
\begin{aligned}
& \operatorname{Tr}\left\{\mathbf{H}_{b l}^{*}\left(\left(\gamma \mathbf{I}_{m}-\widehat{\mathbf{H}}_{l}^{T} \widehat{\mathbf{H}}_{l}\right) \mathbf{H}_{b l}^{* T}+\lambda \widehat{\mathbf{H}}_{l}^{T} \mathbf{S}_{b}\right)\right\} \geq \\
& \operatorname{Tr}\left\{\mathbf{H}_{b l-1}\left(\left(\gamma \mathbf{I}_{m}-\mathbf{H}_{l-1}^{T} \mathbf{H}_{l-1}\right) \mathbf{H}_{b l-1}^{T}+\lambda \mathbf{H}_{l-1}^{T} \mathbf{S}_{b}\right)\right\}
\end{aligned}
$$

where $\widehat{\mathbf{H}}_{l}=\left[\mathbf{H}_{b l}^{*} ; \widetilde{\mathbf{H}}_{l-1}\right]$ and $\mathbf{H}_{l-1}=\left[\mathbf{H}_{b l-1} ; \widetilde{\mathbf{H}}_{l-1}\right]$. Eq. (21) suggests that when $n_{b}<<n$, updating each batch matrix can usually make the objective of Eq. (11) gradually converge to at least a local optima.

\subsection{Time Complexity Analysis}

In Algorithm 1, $n>>d>m$ and $n>>p>m$. Step 1 calculating matrices $\mathbf{A}$ and $\mathbf{H}$ requires $\mathcal{O}(p n d)$ and $\mathcal{O}(n d m)$ operations, respectively. For the outer loop stage, the time complexity of steps 6, 7, 9 and 11 is $\mathcal{O}\left(p m^{2}\right), \mathcal{O}(m), \mathcal{O}\left(p m n_{b}\right)$ and $\mathcal{O}(p m)$, respectively. Hence, the outer loop stage spends $\mathcal{O}\left(L_{1} L_{2}\right.$ npm $)$ operations. Steps 14-16 to calculate the projection matrix A spend $\mathcal{O}\left(p^{2}\right), \mathcal{O}(m)$ and $\max \left(\mathcal{O}(p n m), \mathcal{O}\left(n d^{2}\right)\right)$, respectively. Therefore, the total complexity of Algorithm 1 is $\max \left(\mathcal{O}(n p d), \mathcal{O}\left(n d^{2}\right), \mathcal{O}\left(L_{1} L_{2} n p m\right)\right)$. Empirically, $L_{1} \leq 20$ and $L_{1} \leq 3$.

For Algorithm 2, step 1 calculating $\mathbf{A}, \mathbf{H}$ and $\mathbf{M}$ spends at most $\max \left(\mathcal{O}(p n d), \mathcal{O}\left(n d^{2}\right)\right)$. In the loop stage, the major steps both 7 and 9 require $\mathcal{O}\left(p n_{b}\right)$ operations. Hence, the total time complexity of the loop stage is $\mathcal{O}\left(L_{1} L_{2} n p m\right)$. Additionally, calculating the final $\mathbf{A}$ costs the same time to the steps 14-16 in Algorithm 1. Therefore, the time complexity of Algorithm 2 is $\max \left(\mathcal{O}(n p d), \mathcal{O}\left(n d^{2}\right), \mathcal{O}\left(L_{1} L_{2} n p m\right)\right)$.

\section{Extension to Other Hashing Algorithms}

In this subsection, we illustrate that the proposed algorithm GSDH_P is suitable for solving many other pairwise based hashing models.

Two-step hashing algorithms [14] [42] iteratively update each bit of the different loss functions defined on the Hamming distance of data pairs so that the loss functions of many hashing algorithms such as BRE [8], MLH [10] and EE [41] are incorporated into a general framework, which can be written as:

$$
\min _{\mathbf{h}^{j}} \mathbf{h}^{j} \mathbf{L h}^{j T}, \text { s.t. } \mathbf{h}^{j} \in\{-1,1\}^{n}
$$

where $\mathbf{h}^{j}$ represents the $j$-th bit of binary codes $\mathbf{H} \in$ $\{-1,1\}^{n \times m}$ and $\mathbf{L} \in \mathbb{R}^{n \times n}$ is obtained based on different loss functions with fixing all bits of binary codes except $\mathbf{h}^{j}$.

The algorithms [14] [42] firstly relax $\mathbf{h}^{j} \in\{-1,1\}^{n}$ into $\mathbf{h}^{j} \in[-1,1]^{n}$ and then employ L-BFGS-B [51] to solve the relaxed optimization problem, followed by thresholding to attain the binary vector $\mathbf{h}^{j}$. However, our optimization mechanism can directly solve Eq. (22) without relaxing $\mathbf{h}^{j}$.
Since $\operatorname{Tr}\left(\mathbf{h}^{j} \mathbf{h}^{j T} \mathbf{h}^{j} \mathbf{h}^{j T}\right)=$ const and $\operatorname{Tr}\left(\mathbf{L} \mathbf{L}^{T}\right)=$ const, the problem in Eq. (22) can be equivalently reformulated as:

$$
\min _{\mathbf{h}^{j}}\left\|\mathbf{h}^{j} \mathbf{h}^{j T}-(-\mathbf{L})\right\|_{F}^{2} \text { s.t. } \mathbf{h}^{j} \in\{-1,1\}^{n},
$$

whose optimization type is same as the objective of Eq. (4) w.r.t $\mathbf{H}$. Replacing the constraint $\mathbf{h}^{j} \in\{-1,1\}^{n}$ with $\mathbf{h}^{j}=$ $\operatorname{sgn}\left(\mathbf{X a}_{j}^{T}\right)$, where $\mathbf{a}_{j} \in \mathbb{R}^{d}$ is the $j$-th row vector of $\mathbf{A}$, Eq. (23) becomes:

$$
\min _{\mathbf{a}_{j}}\left\|\mathbf{h}^{j} \mathbf{h}^{j T}-(-\mathbf{L})\right\|_{F}^{2} \text { s.t. } \mathbf{h}^{j}=\operatorname{sgn}\left(\mathbf{X} \mathbf{a}_{j}^{T}\right),
$$

Since $\mathbf{L} \in \mathbb{R}^{n \times n}$ will consume large computation and storage costs for large $n$, we select $p$ training anchors to construct $\mathbf{L}_{A} \in \mathbb{R}^{p \times n}$ based on different loss functions. Replacing $\mathbf{L}$ in Eq. (24) with $\mathbf{L}_{A}$, it becomes:

$$
\min _{\mathbf{a}_{j}}\left\|\mathbf{h}_{A}^{j} \mathbf{h}^{j T}-\left(-\mathbf{L}_{A}\right)\right\|_{F}^{2}, \text { s.t. } \mathbf{h}^{j}=\operatorname{sgn}\left(\mathbf{X} \mathbf{a}_{j}^{T}\right),
$$

Similar to solving Eq. (4), we can firstly obtain $\mathbf{h}^{j}$ and then calculate $\mathbf{a}_{j}$. To attain $\mathbf{h}^{j}$, we still gradually update each batch $\mathbf{h}_{b}^{j}$ by solving the following problem:

$$
\min _{\mathbf{h}_{b}^{j}}\left\|\mathbf{h}_{A}^{j} \mathbf{h}_{b}^{j T}-\left(-\mathbf{L}_{A b}\right)\right\|_{F}^{2}
$$

where $\mathbf{L}_{A b}=\mathbf{L}_{A}(:, i d x) \in \mathbb{R}^{p \times n_{b}}$.

The optimization type of Eq. (26) is the same as that of Eq. (16). $\mathbf{h}^{j}$ can be obtained by gradually updating $\mathbf{h}_{b}^{j}$ as shown in GSDH_P. After obtaining $\mathbf{h}^{j}, \mathbf{a}_{j}$ can be attained by using $\mathbf{a}_{j}=\left(\beta \overline{\mathbf{h}}^{j T}-\mathbf{h}_{A}^{j T} \mathbf{L}_{A}\right) \mathbf{X}\left(\mathbf{X}^{T} \mathbf{X}\right)^{-1}$.

Based on Eqs. (22)-(26), many pairwise based hashing models can lean binary codes by using GSDH_P. For instances, we show the performance on solving the optimization model in BRE [8] [42]:

$$
\mathcal{L}\left(\mathbf{h}_{i}, \mathbf{h}_{j}\right)=\left[m \delta\left(s_{i j}<0\right)-d_{H}\left(\mathbf{h}_{i}, \mathbf{h}_{j}\right)\right]^{2}
$$

where $d_{H}\left(\mathbf{h}_{i}, \mathbf{h}_{j}\right)$ is the Hamming distance between $\mathbf{h}_{i}$ and $\mathbf{h}_{j}$, and $\delta(\cdot) \in\{0,1\}$ is an indicator function. Here, $s_{i j}<0$ denotes $\left(\mathbf{x}_{i}, \mathbf{x}_{j}\right) \in \mathcal{C}$.

One typical model with a hinge loss function [10] [42]:

$\mathcal{L}\left(\mathbf{h}_{i}, \mathbf{h}_{j}\right)=\left\{\begin{array}{cc}{\left[0-d_{H}\left(\mathbf{h}_{i}, \mathbf{h}_{j}\right)\right]^{2}} & \left(\mathbf{x}_{i}, \mathbf{x}_{j}\right) \in \mathcal{M} \\ {\left[\max \left(0.5 m-d_{H}\left(\mathbf{h}_{i}, \mathbf{h}_{j}\right), 0\right)\right]^{2}} & \text { otherwise. }\end{array}\right.$

In this paper, the optimization model in BRE solved by GSDH_P is named as GSDH_P $B R E$. Similarly, the hinge loss function Eq. (28) solved by GSDH_P is named as GSDH_P Hinge.

\section{Experimental Results and Analysis}

We evaluate the proposed algorithms SDH_P and GSDH_P on one benchmark single-label database: CIFAR-10 [21], and two popular multi-label databases: NUS-WIDE [22] and COCO [23]. The CIFAR-10 database contains 60K color images of ten object categories, with each category consisting of $6 \mathrm{~K}$ images. The NUS-WIDE database contains around $270 \mathrm{~K}$ images collected from Flickr, with each image consisting of multiple semantic labels. Totally, this database has 81 ground truth concept labels. Here, similar to [24], 
we choose the images associated with the 21 most frequent labels. In total, there are around $195 \mathrm{~K}$ images. The COCO database consists of about $328 \mathrm{~K}$ images belonging to 91 objects types. We adopt the 2014 training and validation datasets. They have around $83 \mathrm{~K}$ training and $41 \mathrm{~K}$ validation images belonging to 80 object categories.

\subsection{Experimental Setting}

We compare SDH_P and GSDH_P against fifteen stateof-the-art hashing methods including six point-wise and pairwise based algorithms: KSH [13], CCA-ITQ [52], SDH [24], COSDISH [20], KSDH [15], ADGH [18], four ranking algorithms: RSH [32], CGH [30], Top-RSBC [35] and DSeRH [36], and five deep hashing algorithms: CNNH [19], DNNH [37], DHN [45], Hashnet [47] and DCH [48]. Additionally, we also compare BRE [8] and MLH [10] and FastH [42] with GSDH_P ${ }_{B R E}$ and GSDH_P ${ }_{\text {Hinge }}$ to illustrate the generalization of GSDH_P. For KSH and KSDH, we employ the same pairwise matrix as SDH_P and GSDH_P for tackling singlelabel and multi-label tasks. For all deep hashing algorithms, we show their reported retrieval accuracy on each database. Additionally, for better comparing with deep hashing algorithms, we utilize the binary codes learned by GSDH_P as labels to train classification models, by using the AlexNet architecture [53] in the Pytorch framework with pre-trained on the ImageNet database [54], and name this method as GSDH_P*. For SDH_P and GSDH_P, we empirically set $p=1000, n_{b}=100, \beta=10, L_{1}=20$ and $L_{2}=3$.

To evaluate the hashing methods, we utilize three major criteria: MAP, Precision and Recall, to evaluate their ranking performance on the single-label task, and employ two main criteria: NDCG [34] and average cumulative gain (ACG) [32], to assess their performance on multi-label tasks. Given a set of queries, MAP is the mean of the average precision (AP) for each query. AP is defined as:

$$
A P @ R=\frac{\sum_{k=1}^{R} P(k) \delta(k)}{\sum_{k=1}^{R} \delta(k)}
$$

where $R$ is the number of top returned samples, $P(k)$ is the precision at cut-off $k$ in the list, $\delta(k)=1$ if the sample ranked at $k$-th position is relevant, otherwise, $\delta(k)=0$.

NDCG is the normalization of the discounted cumulative gain (DCG), which is calculated by [34] :

$$
D C G=\operatorname{rel}_{1}+\sum_{k=2}^{R} \frac{r e l_{k}}{\log _{2}(k)}
$$

where $k$ is the ranking position and $r e l_{k}$ is the relevance between the $k$-th retrieved sample and the query.

ACG denotes the average cumulative gain, it is defined as:

$$
A C G_{r}=\frac{1}{\left|\mathcal{N}_{r}\right|} \sum_{x \in \mathcal{N}_{r}} r e l_{x}
$$

where $\mathcal{N}_{r}$ represents the retrieved samples within Hamming radius $r$ and $r e l_{x}$ is the relevance between a returned sample $\mathbf{x}$ and the query.

Since all non-deep hashing methods have similar test time, we only show their training time for better comparison. We utilize MATLAB and conduct all experiments on a 3.50GHz Intel Xeon E5-1650 CPU with 128GB memory.
TABLE 1

Ranking performance (MAP) on top 500 retrieved samples and training

\begin{tabular}{|c|c|c|c|c|c|}
\hline \multirow{3}{*}{ Method } & \multicolumn{5}{|c|}{ GIST $(n=10000)$} \\
\hline & \multicolumn{4}{|c|}{ MAP (Top 500) } & Time \\
\hline & 8-bit & 16-bit & 32-bit & 64-bit & 64-bit \\
\hline$\overline{\mathrm{KSH}}$ & 0.4281 & 0.4706 & 0.5098 & 0.5270 & $4.6 \times 10^{3}$ \\
\hline CCA-ITQ & 0.2014 & 0.1984 & 0.2147 & 0.2316 & 0.9 \\
\hline $\mathrm{SDH}$ & 0.4970 & 0.5370 & 0.5670 & 0.5781 & 7.3 \\
\hline COSDISH & 0.5116 & 0.5912 & 0.5980 & 0.6162 & $1.5 \times 10^{1}$ \\
\hline KSDH & 0.5370 & $\overline{0.5740}$ & $\overline{0.5900}$ & $\overline{0.6000}$ & 3.5 \\
\hline ADGH & $\overline{0.5360}$ & 0.5700 & 0.5980 & 0.6020 & 1.4 \\
\hline$\overline{\mathrm{RSH}}$ & 0.2121 & 0.1889 & 0.1812 & 0.1810 & $7.5 \times 10^{4}$ \\
\hline $\mathrm{CGH}$ & 0.3013 & 0.3040 & 0.3255 & 0.3305 & $9.2 \times 10^{2}$ \\
\hline Top-RSBC & 0.2568 & 0.2404 & 0.2400 & 0.2544 & $9.3 \times 10^{4}$ \\
\hline DSeRH & 0.2020 & 0.2070 & 0.2087 & 0.2116 & $2.7 \times 10^{3}$ \\
\hline$\overline{\text { SDH_P }}$ & 0.5755 & 0.5981 & 0.6102 & 0.6222 & $1.1 \times 10^{1}$ \\
\hline GSDH_P & 0.5600 & 0.5992 & 0.6272 & 0.6300 & $2.4 \times 10^{1}$ \\
\hline \multirow{3}{*}{ Method } & \multicolumn{5}{|c|}{$\overline{\text { GIST (Full) }}$} \\
\hline & \multicolumn{4}{|c|}{ MAP (Top 500) } & Time \\
\hline & 8-bit & 16-bit & 32-bit & 64-bit & 64-bit \\
\hline$\overline{\mathrm{KSH}}$ & 0.4057 & 0.4725 & 0.5126 & 0.5317 & $3.5 \times 10^{4}$ \\
\hline CCA-ITQ & 0.2338 & 0.2224 & 0.2473 & 0.2745 & 4.7 \\
\hline SDH & 0.4723 & 0.5700 & 0.5920 & 0.6038 & $5.2 \times 10^{1}$ \\
\hline COSDISH & 0.5593 & 0.6065 & 0.6125 & 0.6255 & $9.2 \times 10^{1}$ \\
\hline$\overline{\mathrm{KSDH}}$ & 0.5687 & 0.5955 & $\overline{0.6015}$ & $\overline{0.6091}$ & $7.6 \times 10^{1}$ \\
\hline ADGH & $\underline{0.5731}$ & $\underline{0.6097}$ & 0.6113 & 0.6119 & 4.4 \\
\hline SDH_P & 0.5735 & 0.6172 & 0.6222 & 0.6279 & $3.3 \times 10^{1}$ \\
\hline GSDH_P & 0.5680 & 0.6142 & 0.6239 & 0.6333 & $6.9 \times 10^{1}$ \\
\hline
\end{tabular}
time (seconds) of different hashing methods on the single-label database CIFAR-10.

TABLE 2

Ranking performance (MAP) on top 1000 retrieved samples and

\begin{tabular}{|c|c|c|c|c|c|}
\hline \multirow{3}{*}{ Method } & \multicolumn{5}{|c|}{ GIST $(n=10000)$} \\
\hline & \multicolumn{4}{|c|}{ MAP (Top 1000) } & Time \\
\hline & 8-bit & 16-bit & 32-bit & 64-bit & 64-bit \\
\hline$\overline{\mathrm{KSH}}$ & 0.4069 & 0.4458 & 0.4908 & 0.5121 & $4.6 \times 10^{3}$ \\
\hline$\overline{B R E}$ & 0.1999 & 0.2028 & 0.2187 & 0.2340 & $1.3 \times 10^{5}$ \\
\hline MLH & 0.1509 & 0.2581 & 0.2491 & 0.2467 & $2.5 \times 10^{2}$ \\
\hline $\mathbf{G S D H} \mathbf{P}_{K S H}$ & 0.5528 & 0.5996 & 0.6126 & 0.6162 & $2.4 \times 10^{1}$ \\
\hline GSDH_P $_{B R E}$ & 0.5478 & 0.5960 & 0.6158 & 0.6251 & $5.8 \times 10^{1}$ \\
\hline GSDH_P $\mathbf{P}_{\text {Hinge }}$ & 0.5582 & 0.5961 & 0.6136 & 0.6240 & $1.2 \times 10^{2}$ \\
\hline FastH $_{K S H}$ & 0.5364 & 0.5814 & 0.6106 & 0.6207 & $4.1 \times 10^{2}$ \\
\hline Fast $_{B R E}$ & 0.5200 & 0.5800 & 0.6104 & 0.6139 & $4.1 \times 10^{2}$ \\
\hline FastH $\mathrm{H}_{\text {Hinge }}$ & 0.5243 & 0.5775 & 0.6150 & 0.6305 & $3.7 \times 10^{2}$ \\
\hline GSDH_P ${ }_{K S H}+\mathrm{DT}$ & 0.5482 & 0.5921 & 0.6207 & 0.6282 & $1.7 \times 10^{2}$ \\
\hline $\mathbf{G S D H}_{-} \mathbf{P}_{B R E}+\mathrm{DT}$ & 0.5221 & 0.5862 & 0.6224 & 0.6292 & $1.8 \times 10^{2}$ \\
\hline GSDH_P ${ }_{\text {Hinge }}+\mathrm{DT}$ & 0.5352 & 0.5972 & 0.6215 & 0.6340 & $2.1 \times 10^{2}$ \\
\hline
\end{tabular}
training time (seconds) of KSH, BRE, MLH and their variants on the CIFAR-10 database.

TABLE 3

Ranking performance (MAP) of GSDH_P* and several popular deep hashing algorithms on the CIFAR-10 database $\left({ }^{\dagger}\right.$ denotes that the shown results are reported in [48]).

\begin{tabular}{|c|c|c|c|c|}
\hline \multirow{2}{*}{ Method } & \multicolumn{4}{|c|}{ MAP @ Top 5000 } \\
\cline { 2 - 5 } & 12-bit & 24-bit & 32-bit & 48-bit \\
\hline CNNH & 0.429 & 0.511 & 0.509 & 0.522 \\
\hline DNNH & 0.552 & 0.566 & 0.558 & 0.581 \\
\hline DHN & $\underline{0.555}$ & $\underline{0.594}$ & $\underline{0.603}$ & $\underline{0.621}$ \\
\hline GSDH_P $^{*}$ & $\mathbf{0 . 7 9 1}$ & $\mathbf{0 . 7 8 6}$ & $\mathbf{0 . 7 8 0}$ & $\mathbf{0 . 7 7 6}$ \\
\hline \hline \multirow{2}{*}{ Method } & \multicolumn{4}{|c|}{ MAP @ H $\leq 2$} \\
\cline { 2 - 5 } & 16 -bit & 32-bit & 48 -bit & 64-bit \\
\hline CNNH $^{\dagger}$ & 0.5512 & 0.5468 & 0.5454 & 0.5364 \\
\hline DNNH $^{\dagger}$ & 0.5703 & 0.5985 & 0.6421 & 0.6118 \\
\hline DHN $^{\dagger}$ & 0.6929 & 0.6445 & 0.5835 & 0.5883 \\
\hline HashNet $^{\dagger}$ & 0.7576 & 0.7776 & 0.6399 & 0.6259 \\
\hline DCH $^{\dagger}$ & $\underline{0.7901}$ & $\underline{0.7979}$ & $\underline{0.8071}$ & $\underline{0.7936}$ \\
\hline GSDH_P $^{*}$ & $\mathbf{0 . 8 4 8 0}$ & $\mathbf{0 . 8 4 8 0}$ & $\mathbf{0 . 8 4 0 0}$ & $\mathbf{0 . 8 3 2 9}$ \\
\hline
\end{tabular}

\subsection{Experiments on CIFAR-10}

We partition the CIFAR-10 database into training and query sets, which consist of $50 \mathrm{~K}$ and $10 \mathrm{~K}$ images, respectively. Each image is aligned and cropped to $32 \times 32$ pixels and 
then represented by a 512-dimensional GIST feature vector [55]. In our experiments, we kernelize GIST feature vectors by using the same kernel type in $\mathrm{KSH}$ [13] and uniformly selecting $1 \mathrm{~K}$ samples from the training set as anchors. Since some comparative multi-wise based algorithms are extremely slow when using a large number of training data, we utilize only a subset of data to train models for the nondeep hashing algorithms: KSH, CCA-ITQ, SDH, COSDISH, KSDH, ADGH, RSH, CGH, Top-RSBC and DSeRH. Similar to $\mathrm{KSH}$, we uniformly pick up $1 \mathrm{~K}$ and 100 images from each category for training and testing, respectively. Then, we evaluate the proposed algorithms and the six non-ranking algorithms (KSH, CCA-ITQ, SDH, COSDISH, KSDH and ADGH) using all training and query images. Their ranking performance in term of MAP with 500 retrieved samples is shown in Table 1. As we can see, when using $10 \mathrm{~K}$ training and $1 \mathrm{~K}$ query images, both SDH_P and GSDH_P achieve better MAPs than the other algorithms at 8-, 16-, 32- and 64bit. The gain of GSDH_P ranges from $1.35 \%$ to $4.88 \%$ over the best competitor except SDH_P. Additionally, GSDH_P obtains higher MAPs than SDH_P at 16-, 32- and 64-bit. When using 50K training and 10K query images, GSDH_P outperforms the other comparative non-deep hashing algorithms except ADCG at 8-bit. Figure 2 presents the precision and recall of various hashing algorithms at 8-, 16-, 32-, 64- and 128-bits on the CIFAR-10 database with Hamming radius being 2. It further demonstrates the superior performance of GSDH_P over the other hashing algorithms. Although SDH_P achieves best precision at 16- and 32-bit, its recall is very low compared to other algorithms.

To illustrate the generation of the proposed algorithm GSDH_P, we uniformly pick up $1 \mathrm{~K}$ and 100 images from each category for training and testing, respectively. We repeat this process 10 times and report the average MAP of KSH, BRE, MLH, FastH and GSDH_P with top 1K samples returned in Table 2. Note that GSDH_P ${ }_{K S H}+\mathrm{DT}$ denotes learning classification models by using decision trees as classifiers and the learned binary codes of GSDH_P $\mathrm{P}_{K S H}$ as labels. Similar definitions for GSDH_P $P_{B R E}+\mathrm{DT}$ and GSDH_P Hinge $_{\text {+DT. Here, we utilize GSDH_P }}$. $K S H$ to represent GSDH_P for a clear comparison. Table 2 illustrates that GSDH_P can achieve significantly better performance than KSH, BRE and MLH. Meanwhile, it also outperforms FastH with lower training costs.

When evaluating the deep hashing algorithms, we follow the experimental protocol in [19], i.e. randomly selecting 500 and 100 images per class for training and testing, respectively. We show the reported MAP of CNNH, DNNH and DHN with $5 \mathrm{~K}$ samples retrieved and the MAP of $\mathrm{CNNH}, \mathrm{DNNH}, \mathrm{DHN}$, HashNet and DCH with Hamming radius being 2 in Table 3, which shows that GSDH_P* significantly outperforms recent state of the arts on the CIFAR-10 database.

\subsection{Experiments on NUS-WIDE}

For the NUS-WIDE database, we partition all images into training and test sets, including around $185 \mathrm{~K}$ training and $10 \mathrm{~K}$ query images, respectively. Each image is represented by the provided 500 Bag-of-Words (BoW) features. In our experiments, similar to KSH [13], we kernelize BoW feature vectors by uniformly selecting $1 \mathrm{~K}$ samples from the training

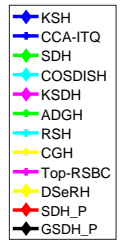

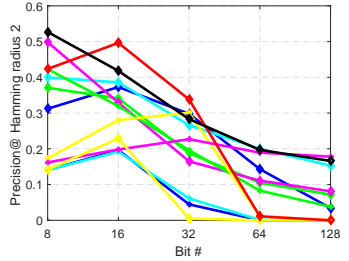

(a) Precision

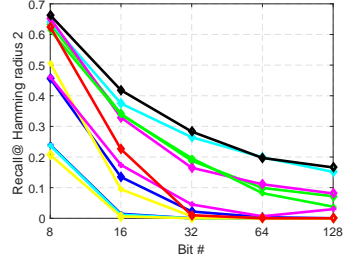

(b) Recall
Fig. 2. Precision and Recall vs. Bits of various algorithms on the CIFAR10 database with Hamming radius being 2 .

TABLE 4

Ranking performance (NDCG) on top 50 retrieved samples and training time (seconds) of different hashing algorithms on the multi-label database NUS-WIDE.

\begin{tabular}{|c|c|c|c|c|c|}
\hline \multirow{3}{*}{ Method } & \multicolumn{5}{|c|}{$\mathrm{BoW}(n=10000)$} \\
\hline & \multicolumn{4}{|c|}{ NDCG (Top 50) } & Time \\
\hline & 8-bit & 16-bit & 32-bit & 64-bit & 64-bit \\
\hline KSH & 0.2755 & 0.2730 & 0.2554 & 0.2395 & $2.1 \times 10^{3}$ \\
\hline CCA-ITQ & 0.2522 & 0.2337 & 0.2248 & 0.2145 & 0.4 \\
\hline$\overline{\mathrm{SDH}}$ & 0.3108 & $\underline{0.3674}$ & 0.3674 & 0.3494 & $2.5 \times 10^{\mathrm{I}}$ \\
\hline COSDISH & 0.3169 & 0.3606 & 0.3989 & 0.3801 & $3.5 \times 10^{1}$ \\
\hline KSDH & 0.3227 & 0.3125 & 0.3091 & 0.3092 & 5.3 \\
\hline$\overline{\mathrm{RSH}}$ & 0.2040 & 0.2061 & 0.2088 & 0.2193 & $9.2 \times 10^{4}$ \\
\hline CGH & 0.2163 & 0.2228 & 0.2475 & 0.2323 & $1.5 \times 10^{3}$ \\
\hline Top-RSBC & 0.1962 & 0.2238 & 0.2564 & 0.2758 & $8.9 \times 10^{4}$ \\
\hline DSeRH & 0.2158 & 0.2264 & 0.2271 & 0.2210 & $8.2 \times 10^{3}$ \\
\hline SDH_P & 0.2916 & 0.3014 & 0.3038 & 0.3174 & 4.3 \\
\hline GSDH_P & 0.3588 & 0.3889 & 0.4083 & 0.4311 & $5.5 \times 10^{\perp}$ \\
\hline \multirow{3}{*}{ Method } & \multicolumn{5}{|c|}{$\overline{\text { BoW (Full) }}$} \\
\hline & \multicolumn{4}{|c|}{ NDCG (Top 50) } & Time \\
\hline & 8-bit & 16-bit & 32-bit & 64-bit & 64-bit \\
\hline CCA-ITQ & 0.2562 & 0.2551 & 0.2528 & 0.2475 & 8.8 \\
\hline$\overline{\mathrm{SDH}}$ & 0.3381 & $\underline{0.3927}$ & 0.3838 & 0.3827 & $3.8 \times 10^{2}$ \\
\hline COSDISH & $\underline{0.3649}$ & 0.3539 & $\underline{0.4279}$ & $\underline{0.4323}$ & $3.5 \times 10^{2}$ \\
\hline SDH_P & 0.3010 & 0.3094 & 0.3431 & 0.3313 & $7.7 \times 10^{1}$ \\
\hline GSDH_P & 0.4152 & 0.4521 & 0.4646 & .4875 & $5.0 \times 10^{2}$ \\
\hline
\end{tabular}

TABLE 5

Ranking performance (MAP) of GSDH_P* and several popular deep hashing algorithms on the NUSWIDE database $\left({ }^{\dagger}\right.$ denotes that the shown results are reported in [48]).

\begin{tabular}{|c|c|c|c|c|}
\hline \multirow{2}{*}{ Method } & \multicolumn{4}{|c|}{ MAP @ Top 5000 } \\
\cline { 2 - 5 } & 12-bit & 24-bit & 32-bit & 48-bit \\
\hline CNNH & 0.617 & 0.663 & 0.657 & 0.688 \\
\hline DNNH & 0.674 & 0.697 & 0.713 & 0.715 \\
\hline DHN & $\underline{0.708}$ & $\underline{0.735}$ & $\underline{0.748}$ & $\underline{0.758}$ \\
\hline GSDH_P $^{*}$ & $\mathbf{0 . 7 5 9}$ & $\mathbf{0 . 7 9 1}$ & $\mathbf{0 . 8 0 6}$ & $\mathbf{0 . 7 9 9}$ \\
\hline \hline \multirow{2}{*}{ Method } & \multicolumn{4}{|c|}{ MAP @ H $\leq$ H } \\
\cline { 2 - 5 } & 16 -bit & 32-bit & 48 -bit & 64-bit \\
\hline CNNH $^{\dagger}$ & 0.5843 & 0.5989 & 0.5734 & 0.5729 \\
\hline DNNH $^{\dagger}$ & 0.6191 & 0.6216 & 0.5902 & 0.5626 \\
\hline DHN $^{\dagger}$ & 0.6901 & 0.7021 & 0.6736 & 0.6190 \\
\hline HashNet $^{\dagger}$ & 0.6944 & 0.7147 & 0.6736 & 0.6190 \\
\hline DCH $^{\dagger}$ & $\underline{0.7401}$ & $\underline{0.7720}$ & $\underline{0.7685}$ & $\underline{0.7124}$ \\
\hline GSDH_P $^{*}$ & $\mathbf{0 . 8 0 7 3}$ & $\mathbf{0 . 8 0 2 5}$ & 0.7572 & $\mathbf{0 . 7 4 4 2}$ \\
\hline
\end{tabular}

set as anchors. Firstly, we uniformly select $10 \mathrm{~K}$ training and $1 \mathrm{~K}$ query images to evaluate all ranking and non-ranking algorithms except ADGH, since it cannot be directly applied to tackling multi-label tasks. Then, we utilize all training and query images to evaluate the proposed algorithms and three scalable algorithms: CCA-ITQ, SDH and COSDISH. Table 4 presents their ranking performance in term of NDCG with 50 samples retrieved. It shows that GSDH_P has superior ranking performance to the other nine nondeep hashing algorithms when $10 \mathrm{~K}$ training images are used. Its gain in term of NDCG is from $2.35 \%$ to $13.42 \%$ over the best competitors except SDH_P. When all training images are used, GSDH_P significantly outperforms CCAITQ, SDH and COSDISH, its gain ranges from $8.58 \%$ to 

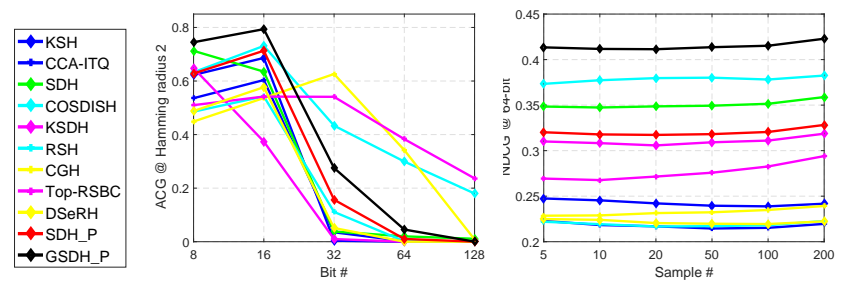

(a) ACG

(b) NDCG

Fig. 3. ACG vs. Bits and NDCG vs. retrieved samples of various algorithms with images selected from the NUS-WIDE database.

TABLE 6

Ranking performance (NDCG) on top 50 retrieved samples and training time (seconds) of various hashing algorithms on images from the multi-label database COCO.

\begin{tabular}{|c|c|c|c|c|c|}
\hline \multirow{3}{*}{ Method } & \multicolumn{5}{|c|}{$n=10000$} \\
\hline & \multicolumn{4}{|c|}{ NDCG (Top 50) } & Time \\
\hline & 8-bit & 16-bit & 32-bit & 64-bit & 64-bit \\
\hline KSH & 0.2352 & 0.3226 & 0.3810 & 0.4168 & $2.7 \times 10^{3}$ \\
\hline CCA-ITQ & $\overline{0.2293}$ & 0.3367 & 0.3947 & 0.4103 & 0.3 \\
\hline $\mathrm{SDH}$ & 1911 & $\overline{0.3218}$ & $\overline{0.3911}$ & 0.4345 & $2.1 \times 10^{1}$ \\
\hline COSDIS & 1623 & 0.2382 & 0.2438 & 0.2890 & $2.2 \times 10^{1}$ \\
\hline KSDH & 0.1623 & 0.2000 & 0.2292 & 0.2597 & 2.7 \\
\hline$\overline{\mathrm{RSH}}$ & 0.2156 & $\overline{0.2751}$ & 0.3299 & $\overline{0.3583}$ & $6.1 \times 10^{4}$ \\
\hline CGH & 566 & 0.1928 & 0.2176 & 0.2324 & $1.9 \times 10^{3}$ \\
\hline Top-RSBC & 1547 & 0.1843 & 0.2045 & 0.2845 & $8.6 \times 10^{4}$ \\
\hline DSeRH & 0.2144 & 0.2556 & 0.3210 & 0.3560 & $1.7 \times 10^{3}$ \\
\hline SDH_P & 0.1932 & 0.2476 & 0.3165 & 0.3465 & 2.7 \\
\hline GSDH_P & 0.2526 & 0.3550 & 0.4136 & 0.4385 & $5.5 \times 10^{1}$ \\
\hline & \multicolumn{5}{|c|}{$\overline{\text { Full }}$} \\
\hline & \multicolumn{4}{|c|}{ NDCG (Top 50) } & Time \\
\hline & 8-bit & 16-bit & 32-bit & 64-bit & 64-bit \\
\hline CCA-ITQ & 0.1831 & 0.2426 & 0.2902 & 0.3032 & $1.0 \times 10^{\perp}$ \\
\hline SDH & $\overline{0.1684}$ & 0.2430 & 0.3537 & 0.3846 & $3.2 \times 10^{2}$ \\
\hline COSDISH & 0.1557 & 0.1741 & 0.2013 & 0.2146 & $1.9 \times 10^{2}$ \\
\hline SDH_P & 0.1756 & 0.2427 & 0.3252 & 0.3471 & $2.3 \times 10^{1}$ \\
\hline$\overline{\text { GSDH_P }}$ & .2240 & 0.3160 & 0.3760 & 0.4020 & $6.1 \times 10^{2}$ \\
\hline
\end{tabular}

$15.13 \%$ over the best competitors at all four bits. Figure 3 presents the ACG of various algorithms on the NUS-WIDE database at 8-, 16-, 32-, 64- and 128-bit with Hamming radius being 2, and their NDCGs with 5, 10, 20, 50, 100 and 200 samples retrieved at 64-bit. It further illustrates that GSDH_P outperforms the other hashing algorithms.

Additionally, we follow the protocol in [19] [37] to randomly select 100 query and uniformly sample 500 training images from each of the selected 21 most frequent labels, in order to evaluate the MAP of GSDH_P* and deep hashing algorithms $\mathrm{CNNH}, \mathrm{DNNH}$ and DHN with top 5000 images returned. Moreover, to evaluate the MAP of GSDH_P*, $\mathrm{CNNH}, \mathrm{DNNH}, \mathrm{DHN}$, HashNet and DCH with Hamming radius being 2, we follow the experimental protocol in $\mathrm{DCH}$ [48], i.e. randomly sample $5 \mathrm{~K}$ and $10 \mathrm{~K}$ images to construct testing and training sets, respectively. Note that when calculating MAP, if two images share at least one label, they are similar and $s_{i j}=1$, otherwise, they are dissimilar and $s_{i j}=0$. Table 5 shows their MAP with top 5K retrieved samples and Hamming radius being 2. It figures out that GSDH_P* can significantly outperform $\mathrm{CNNH}$, DNNH and DHN when top 5K images are returned, and when Hamming radius being 2, GSDH_P* achieves 6.72\%, $3.05 \%$ and $3.18 \%$ higher MAP than the best competitor DCH at 16-, 32- and 64-bit.

\subsection{Experiments on COCO}

For the COCO database, we adopt $83 \mathrm{~K}$ training images and select $10 \mathrm{~K}$ validation images to construct training and query

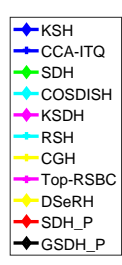

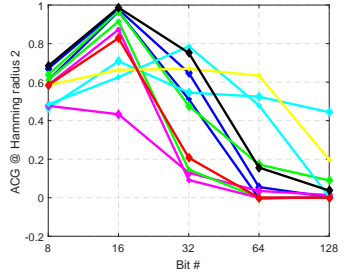

(a) $\mathrm{COCO}$

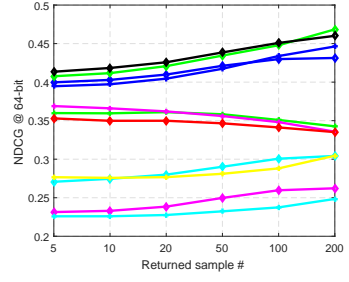

(b) $\mathrm{COCO}$
Fig. 4. ACG vs. Bits and NDCG vs. retrieved samples of various algorithms with images selected from the NUS-WIDE database.

sets, respectively. Then we resize each image to $32 \times 32$ pixels and represent each one by using a 2048-dimensional CNN feature vector, which is extracted by a popular and powerful neural network: ResNet50 [56]. After that, we kernelize the features by using the same kernel type as KSH with 1000 anchors selected. Firstly, we uniformly select $10 \mathrm{~K}$ training and $1 \mathrm{~K}$ query images to evaluate the proposed algorithms SDH_P and GSDH_P, and nine non-deep hashing algorithms. Then we adopt all training and query images to evaluate the scalable hashing algorithms CCA-ITQ, SDH, COSDISH, SDH_P and GSDH_P.

Table 6 displays their ranking performance in term of NDCG with top 50 retrieved samples and training time of various algorithms on the COCO database. It illustrates that GSDH_P has the superior performance to the other hashing algorithms. When using $10 \mathrm{~K}$ training images, the gain of GSDH_P in term of NDCG is $7.40 \%, 5.44 \%, 4.79 \%$ and $0.92 \%$ compared to the best competitor except SDH_P at 8-, 16-, 32- and 64-bit, respectively; when using all training images, the NDCG of GSDH_P is $4.09 \%, 7.30 \%, 2.23 \%$ and $1.74 \%$ higher than the best competitor except SDH_P at the four bits, respectively. Figure 4 presents the ACG of various algorithms on the COCO database at 8-, 16-, 32-, 64- and 128-bit with Hamming radius being 2, and presents their NDCGs with 5, 10, 20, 50, 100 and 200 samples retrieved at 64-bit. It further demonstrates the strength of GSDH_P.

\subsection{Parameter Influence}

Here, we mainly evaluate two essential parameters: batch size $n_{b}$ and regularization parameter $\beta$, where $n_{b}$ and $\beta$ determine the batch number $f$ and the parameter $\gamma$, respectively. Similar to previous experiments, we uniformly select $10 \mathrm{~K}$ training and $1 \mathrm{~K}$ query images from CIFAR-10, NUS-WIDE and COCO databases, and then encode each image features into 16-bit binary codes. Figure 5 shows the influence of $n_{b} \in\left[1,10,10^{2}, 10^{3}, 5 \times 10^{3}, 10^{4}\right]$ and $\beta \in\left[0,1,10,10^{2}, 10^{3}, 10^{4}, 10^{5}\right]$ on ranking performance in term of MAP and NDCG on the three databases. It suggests that both SDH_P and GSDH_P can obtain the best or sub-best ranking performance when $n_{b} \in[1,100]$ and $\beta \in[0,100]$ on all the three databases. Similar results can be found at other bits, we do not show them for brevity. In our single-label and multi-label experiments, without loss of generality, we empirically set $\beta=10$ and $n_{b}=100$ for the proposed algorithms.

\section{Conclusion}

In this paper, we propose a novel, scalable and general optimization method to directly solve the non-convex and non-smooth problems in term of hash functions. We firstly 


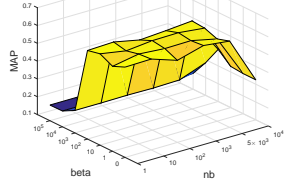

(a) CIFAR@ SDH_P

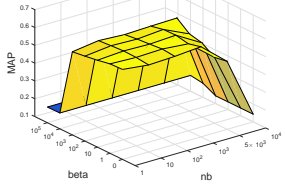

(b) CIFAR@ GSDH_P

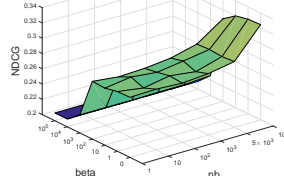

(c) NUS@SDH_P

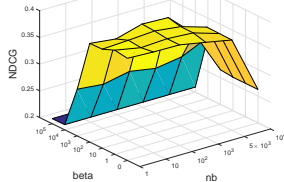

(d) NUS@ GSDH_P

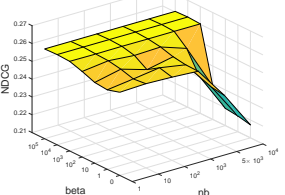

(e) COCO@ SDH_P

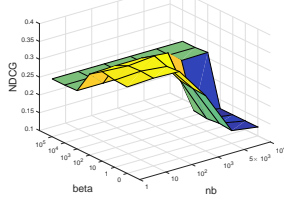

(f) COCO@ GSDH_P

Fig. 5. The influence of parameters $n_{b}$ and $\beta$ for SDH_P and GSDH_P on CIFAR-10, NUSWIDE and COCO databases.

solve a quartic problem in a least-squares model that utilizes two identical code matrices produced by hash functions to approximate a pairwise label matrix, by reformulating the quartic problem in term of hash functions into a quadratic problem, and then linearize it by introducing a linear regression model. Additionally, we find that gradually learning each batch of binary codes is beneficial to the convergence of learning process. Based on this finding, we propose a symmetric discrete hashing algorithm to gradually update each batch of the discrete matrix, and a greedy symmetric discrete hashing algorithm to greedily update each bit of batch discrete matrices. Finally, we extend the proposed greedy symmetric discrete hashing algorithm to handle other optimization problems. Extensive experiments on single-label and multi-label databases demonstrate the effectiveness and efficiency of the proposed method. In this paper we only focus on solving the problems in pairwise based hashing, in the future, it is worth extending the proposed mechanism to solve the problems in multi-wise based hashing, whose objective is also highly non-differential, non-convex and more difficult to directly solve.

\section{REFERENCES}

[1] A. Torralba, R. Fergus, and Y. Weiss, "Small codes and large image databases for recognition," in Proceedings of the IEEE Conference on Computer Vision and Pattern Recognition, 2008, pp. 1-8.

[2] W. Liu, C. Mu, S. Kumar, and S.-F. Chang, "Discrete graph hashing," in Advances in Neural Information Processing Systems, 2014, pp. 3419-3427.

[3] Y. Cao, H. Qi, W. Zhou, J. Kato, K. Li, X. Liu, and J. Gui, “Binary hashing for approximate nearest neighbor search on big data: A survey," IEEE Access, vol. 6, pp. 2039-2054, 2018.

[4] P. Indyk and R. Motwani, "Approximate nearest neighbors: towards removing the curse of dimensionality," in Proceedings of the ACM symposium on Theory of computing, 1998, pp. 604-613.

[5] A. Z. Broder, M. Charikar, A. M. Frieze, and M. Mitzenmacher, "Min-wise independent permutations," Journal of Computer and System Sciences, vol. 60, no. 3, pp. 630-659, 2000.

[6] A. Shrivastava and P. Li, "In defense of minhash over simhash," in Artificial Intelligence and Statistics, 2014, pp. 886-894.

[7] Y. Weiss, A. Torralba, and R. Fergus, "Spectral hashing," in Advances in Neural Information Processing Systems, 2009, pp. 1753-1760.

[8] B. Kulis and T. Darrell, "Learning to hash with binary reconstructive embeddings," in Advances in Neural Information Processing Systems, 2009, pp. 1042-1050.

[9] Y. Gong, S. Lazebnik, A. Gordo, and F. Perronnin, "Iterative quantization: A procrustean approach to learning binary codes for large-scale image retrieval," IEEE Transactions on Pattern Analysis and Machine Intelligence, vol. 35, no. 12, pp. 2916-2929, 2013.

[10] M. Norouzi and D. M. Blei, "Minimal loss hashing for compact binary codes," in Proceedings of International Conference on Machine Learning, 2011, pp. 353-360.

[11] J. Wang, S. Kumar, and S.-F. Chang, "Semi-supervised hashing for large-scale search," IEEE Transactions on Pattern Analysis and Machine Intelligence, vol. 34, no. 12, pp. 2393-2406, 2012.

[12] C. Strecha, A. Bronstein, M. Bronstein, and P. Fua, "Ldahash: Improved matching with smaller descriptors," IEEE Transactions on Pattern Analysis and Machine Intelligence, vol. 34, no. 1, pp. 6678, 2012.
[13] W. Liu, J. Wang, R. Ji, Y.-G. Jiang, and S.-F. Chang, "Supervised hashing with kernels," in Proceedings of the IEEE Conference on Computer Vision and Pattern Recognition, 2012, pp. 2074-2081.

[14] G. Lin, C. Shen, D. Suter, and A. van den Hengel, "A general two-step approach to learning-based hashing," in Proceedings of the IEEE International Conference on Computer Vision, 2013, pp. 25522559.

[15] X. Shi, F. Xing, J. Cai, Z. Zhang, Y. Xie, and L. Yang, "Kernel-based supervised discrete hashing for image retrieval," in European Conference on Computer Vision, 2016, pp. 419-433.

[16] B. Neyshabur, N. Srebro, R. R. Salakhutdinov, Y. Makarychev, and P. Yadollahpour, "The power of asymmetry in binary hashing," in Advances in Neural Information Processing Systems, 2013, pp. 28232831.

[17] F. Shen, W. Liu, S. Zhang, Y. Yang, and H. T. Shen, "Learning binary codes for maximum inner product search," in Proceedings of the IEEE International Conference on Computer Vision, 2015, pp. $4148-4156$.

[18] X. Shi, F. Xing, K. Xu, M. Sapkota, and L. Yang, "Asymmetric discrete graph hashing," in AAAI Conference on Artificial Intelligence, 2017, pp. 2541-2547.

[19] R. Xia, Y. Pan, H. Lai, C. Liu, and S. Yan, "Supervised hashing for image retrieval via image representation learning." in $A A A I$ Conference on Artificial Intelligence, vol. 1, 2014, pp. 2156-2162.

[20] W.-C. Kang, W.-J. Li, and Z.-H. Zhou, "Column sampling based discrete supervised hashing," in AAAI Conference on Artificial Intelligence, 2016.

[21] A. Torralba, R. Fergus, and W. T. Freeman, "80 million tiny images: A large data set for nonparametric object and scene recognition," IEEE Transactions on Pattern Analysis and Machine Intelligence, vol. 30, no. 11, pp. 1958-1970, 2008.

[22] T.-S. Chua, J. Tang, R. Hong, H. Li, Z. Luo, and Y. Zheng, "Nuswide: a real-world web image database from national university of singapore," in Proceedings of the ACM International Conference on Image and Video Retrieval, 2009, p. 48.

[23] T.-Y. Lin, M. Maire, S. Belongie, J. Hays, P. Perona, D. Ramanan, P. Dollar, and C. L. Zitnick, "Microsoft coco: Common objects in context," in ECCV. Springer, 2014, pp. 740-755.

[24] F. Shen, C. Shen, W. Liu, and H. Tao Shen, "Supervised discrete hashing," in Proceedings of the IEEE Conference on Computer Vision and Pattern Recognition, 2015, pp. 37-45.

[25] G. Koutaki, K. Shirai, and M. Ambai, "Fast supervised discrete hashing and its analysis," arXiv preprint arXiv:1611.10017, 2016.

[26] X. Wang, T. Zhang, G.-J. Qi, J. Tang, and J. Wang, "Supervised quantization for similarity search," in Proceedings of the IEEE Conference on Computer Vision and Pattern Recognition, 2016, pp. 2018-2026.

[27] K. Lin, H.-F. Yang, J.-H. Hsiao, and C.-S. Chen, “Deep learning of binary hash codes for fast image retrieval," in Proceedings of the IEEE Conference on Computer Vision and Pattern Recognition Workshops (CVPRW), 2015, pp. 27-35.

[28] M. Sapkota, X. Shi, F. Xing, and L. Yang, "Deep convolutional hashing for low dimensional binary embedding of histopathological images," IEEE Journal of Biomedical and Health Informatics, 2018.

[29] M. Norouzi, D. J. Fleet, and R. R. Salakhutdinov, "Hamming distance metric learning," in Advances in Neural Information Processing Systems, 2012, pp. 1061-1069.

[30] X. Li, G. Lin, C. Shen, A. Hengel, and A. Dick, "Learning hash functions using column generation," in Proceedings of International Conference on Machine Learning, 2013, pp. 142-150.

[31] J. Wang, J. Wang, N. Yu, and S. Li, "Order preserving hashing for approximate nearest neighbor search," in ACM MM, 2013, pp. 133-142.

[32] J. Wang, W. Liu, A. X. Sun, and Y.-G. Jiang, "Learning hash codes with listwise supervision," in Proceedings of the IEEE International Conference on Computer Vision, 2013, pp. 3032-3039. 
[33] Q. Wang, Z. Zhang, and L. Si, "Ranking preserving hashing for fast similarity search," in International Joint Conference on Artificial Intelligence, 2015, pp. 3911-3917.

[34] K. Járvelin and J. Kekäläinen, "Ir evaluation methods for retrieving highly relevant documents," in Proceedings of ACM SIGIR Conference on Research \& Development in Information Retrieval. ACM, 2000, pp. 41-48.

[35] D. Song, W. Liu, R. Ji, D. A. Meyer, and J. R. Smith, “Top rank supervised binary coding for visual search," in Proceedings of the IEEE Conference on Computer Vision, 2015, pp. 1922-1930.

[36] L. Liu, L. Shao, F. Shen, and M. Yu, "Discretely coding semantic rank orders for supervised image hashing," in Proceedings of the IEEE Conference on Computer Vision and Pattern Recognition, 2017, pp. 1425-1434.

[37] H. Lai, Y. Pan, Y. Liu, and S. Yan, "Simultaneous feature learning and hash coding with deep neural networks," in Proceedings of the IEEE Conference on Computer Vision and Pattern Recognition, 2015, pp. 3270-3278.

[38] F. Zhao, Y. Huang, L. Wang, and T. Tan, "Deep semantic ranking based hashing for multi-label image retrieval," in Proceedings of the IEEE Conference on Computer Vision and Pattern Recognition, 2015, pp. 1556-1564.

[39] B. Zhuang, G. Lin, C. Shen, and I. Reid, "Fast training of tripletbased deep binary embedding networks," in Proceedings of the IEEE Conference on Computer Vision and Pattern Recognition, 2016, pp. 5955-5964.

[40] W. Liu, J. Wang, S. Kumar, and S.-F. Chang, "Hashing with graphs," in Proceedings of International Conference on Machine Learning, 2011, pp. 1-8.

[41] M. A. Carreira-Perpinán, "The elastic embedding algorithm for dimensionality reduction," in Proceedings of International Conference on Machine Learning, vol. 10, 2010, pp. 167-174.

[42] G. Lin, C. Shen, and A. van den Hengel, "Supervised hashing using graph cuts and boosted decision trees," IEEE Transactions on Pattern Analysis and Machine Intelligence, vol. 37, no. 11, pp. 23172331, 2015.

[43] P. Zhang, W. Zhang, W.-J. Li, and M. Guo, "Supervised hashing with latent factor models," in Proceedings of ACM SIGIR Conference on Research $\mathcal{E}$ Development in Information Retrieval, 2014, pp. 173182.

[44] D. Zhang, J. Wang, D. Cai, and J. Lu, "Self-taught hashing for fast similarity search," in Proceedings of ACM SIGIR Conference on Research and Development in Information Retrieval, 2010, pp. 18-25.

[45] H. Zhu, M. Long, J. Wang, and Y. Cao, "Deep hashing network for efficient similarity retrieval." in AAAI Conference on Artificial Intelligence, 2016, pp. 2415-2421.

[46] W.-J. Li, S. Wang, and W.-C. Kang, "Feature learning based deep supervised hashing with pairwise labels," arXiv preprint arXiv:1511.03855, 2015.

[47] Z. Cao, M. Long, J. Wang, and S. Y. Philip, "Hashnet: Deep learning to hash by continuation." in Proceedings of the IEEE Conference on Computer Vision, 2017, pp. 5609-5618.

[48] Y. Cao, M. Long, B. Liu, J. Wang, and M. KLiss, “Deep cauchy hashing for hamming space retrieval," in Proceedings of the IEEE Conference on Computer Vision and Pattern Recognition, 2018, pp. 1229-1237.

[49] X. Shi, M. Sapkota, F. Xing, F. Liu, L. Cui, and L. Yang, "Pairwise based deep ranking hashing for histopathology image classification and retrieval," Pattern Recognition, vol. 81, pp. 14-22, 2018.

[50] G. Lin, C. Shen, Q. Shi, A. van den Hengel, and D. Suter, "Fast supervised hashing with decision trees for high-dimensional data," in Proceedings of the IEEE Conference on Computer Vision and Pattern Recognition, 2014, pp. 1963-1970.

[51] C. Zhu, R. H. Byrd, P. Lu, and J. Nocedal, "Algorithm 778: L-bfgs-b: Fortran subroutines for large-scale bound-constrained optimization," ACM Transactions on Mathematical Software, vol. 23, no. 4, pp. 550-560, 1997.

[52] Y. Gong, S. Lazebnik, A. Gordo, and F. Perronnin, "Iterative quantization: A procrustean approach to learning binary codes for large-scale image retrieval," IEEE Transactions on Pattern Analysis and Machine Intelligence, vol. 35, no. 12, pp. 2916-2929, 2013.

[53] A. Krizhevsky, I. Sutskever, and G. E. Hinton, "Imagenet classification with deep convolutional neural networks," in Advances in Neural Information Processing Systems, 2012, pp. 1097-1105.

[54] J. Deng, W. Dong, R. Socher, L.-J. Li, K. Li, and L. Fei-Fei, "Imagenet: A large-scale hierarchical image database," in Proceedings of the IEEE Conference on Computer Vision and Pattern Recognition, 2009, pp. 248-255.

[55] A. Oliva and A. Torralba, "Modeling the shape of the scene: A holistic representation of the spatial envelope," International Journal of Computer Vision, vol. 42, no. 3, pp. 145-175, 2001.

[56] K. He, X. Zhang, S. Ren, and J. Sun, "Deep residual learning for image recognition," in Proceedings of the IEEE Conference on Computer Vision and Pattern Recognition, 2016, pp. 770-778. 